\title{
Parabolic and white-noise approximations for elastic waves in random media
}

\author{
Josselin Garnier \\ Laboratoire de Probabilités et Modèles Aléatoires 83 Laboratoire Jacques-Louis Lions, Université Paris VII, 2 Place Jussieu, \\ 75251 Paris Cedex 05, France \\ Knut Sølna \\ Department of Mathematics, University of California, Irvine CA 92697
}

\begin{abstract}
In this paper we carry out an asymptotic analysis of the elastic wave equations in random media in the parabolic white-noise regime. In this regime, the propagation distance is much larger than the initial beam width, which is itself much larger than the typical wavelength; moreover, the correlation length of the random medium is of the same order as the initial beam width, and the amplitude of the random fluctuations is small. In this distinguished limit we show that wave propagation is governed by a system of random paraxial wave equations. The equations for the shear waves and pressure waves have the form of Schrödinger equations driven by two correlated Brownian fields. The diffraction operators can be expressed in terms of transverse Laplacians. The covariance structure of the Brownian fields is determined by the two point-statistics of the density and Lamé parameters of the random medium.
\end{abstract}

\section{Introduction}

The parabolic approximation for wave propagation in heterogeneous media is a model used in a vast number of applications, for instance in communication and imaging [31]. It usually has the form of a Schrödinger equation that describes waves propagating along a privileged propagation axis determined by the source. It is very simple compared to the full three-dimensional wave equation, both from the theoretical and numerical viewpoints, and it enables analysis of many important phenomena, such as laser beam propagation [30], time reversal in random media [4,26], underwater acoustics [31] or migration problems in geophysics [5].

The parabolic approximation for scalar waves in homogeneous and random media is rather well understood. It is even valid in some scaling regimes in which the medium fluctuations are rapid and can be approximated by a white-noise term. The main motivations for studying the white-noise paraxial wave equation are (i) it appears as a very natural model in many situations where the correlation length of the medium is relatively small, in particular much smaller than the propagation distance, (ii) it allows for the use of Itô's stochastic calculus, which in turn enables the closure of the hierarchy of moment equations and the statistical analysis of important wave propagation problems, such as scintillation [12]. When the paraxial approximation and the white-noise approximation can be justified simultaneously for scalar waves,

Email addresses: garnier@math.jussieu.fr (Josselin Garnier), ksolna@math.uci.edu (Knut Sølna). 
then the limit equation takes the form of the random Schrödinger equation studied in particular in [7]. The proof of the convergence of the solution of the wave equation in random media to the solution of the white-noise paraxial equation was obtained for stratified weakly fluctuating media in [2] and recently for three-dimensional random media in the context of acoustic waves in [14].

A parabolic approximation for linear elastic waves is of great interest for solving migration problems in geophysics [8]. In the theory of elastic waves the field is described by the displacement vector. It is not straightforward to extend the parabolic approximation to elastic waves because two different wavenumbers coexist for plane waves in a given direction in a homogeneous medium, one for pressure waves and one for shear waves. The effective behavior of the coupling terms between shear waves and pressure waves in heterogeneous media is not easy to incorporate in the parabolic approximation. The analysis of the effective coupling is one of the main objectives of this work. In this paper we consider three-dimensional linear elastic waves. Motivated by problems in geophysics, we assume that the privileged propagation axis is the vertical direction. This choice explains the terminology "downward-going" waves and "upward-going" waves that we use in the context of the wave decomposition that we set forth.

In the literature it is possible to find parabolic approximation models for the linear elastic wave equations which are obtained by suppressing the reflection terms. In [22] this idea is used to transform the secondorder boundary value problem for two-dimensional elastic waves in laterally inhomogeneous media into a first-order initial value problem in space, whose numerical solutions can be computed efficiently. In [25] a parabolic approximation is constructed for three-dimensional elastic waves where the wave field disturbance is characterized by two almost plane waves travelling with wave speeds associated with pressure and shear waves. In [18] the author uses a multi-scale expansion and deals separately with the shear waves and the pressure waves. In [6] the reflection terms are suppressed based on the argument that the phases of the coupling terms between the downward-going and the upward-going waves vary rapidly making these terms negligible in the lowest order approximation. All these derivations are formal and give quantitatively different predictions [33].

Our goal is to provide a rigorous derivation of the paraxial wave equations for elastic waves in homogeneous media and, moreover, in random media.

1) In the case of a homogeneous medium, we consider the distinguished limit in which the propagation distance is much larger than the initial beam width, which is itself much larger than the typical wavelength. We apply an invariant imbedding theorem and an averaging theorem for rapidly oscillating differential equations in order to prove the convergence of the solution of the wave equation to the solution of a system of Schrödinger equations. The invariant imbedding allows us to transform the boundary value problem into an initial value problem. The averaging theorem allows us to derive effective paraxial wave equations for elastic waves. In particular, we find that the coupling terms between downward-going waves and upwardgoing waves, and between shear waves and pressure waves, have rapid phases. By using an averaging theorem for highly oscillatory differential equations, we show that these coupling terms average out to give non-zero effective terms (in the form of Lie brackets) for downward-going shear waves and for the downward-going pressure waves. There is no effective energy transfer between the downward-going waves and the upwardgoing waves, neither between pressure waves and shear waves. However, the effective or implicit coupling between the wave modes affects the propagation of the downward-going shear waves and the one of the downward-going pressure waves. It is therefore important to take into account these effects. It turns out that the explicit computation of the effective Lie brackets gives very simple forms for the parabolic approximations of the pressure waves and the shear waves.

2) In the case of a random medium, we assume additionally that the correlation length of the random medium is of the same order as the initial beam width, and that the amplitude of the random fluctuations is small. By applying diffusion approximation theorems we prove the convergence of the solution of the random elastic wave equations to the solution of a system of Itô-Schrödinger equations. These Schrödinger equations are driven by two correlated Brownian fields, whose covariance function depends on the two-point statistics of the fluctuations of the density and Lamé parameters of the medium. This result shows that it is possible to justify both the parabolic approximation and the white-noise approximation in the distinguished limit considered in this paper. The limit system permits easy numerical simulations on the one hand, and theoretical computations of moments using Itô's formula on the other hand. 
The paper is organized as follows. In Section 2 we describe the linear elastic wave equations in a random medium. We then introduce the fundamental wave decomposition that we use in the analysis in Section 3 . Next we present the main results, the random paraxial wave equations, in Section 4 . The proofs of the results are given in Section 5 when the medium is homogeneous and in Section 6 when the medium is randomly heterogeneous.

\section{Elastic waves in a random medium}

We consider linear elastic waves propagating in a three-dimensional medium with heterogeneous and random fluctuations. The equations of motion for small displacements $\boldsymbol{u}(t, \boldsymbol{x})=\left(u_{j}(t, \boldsymbol{x})\right)_{j=1,2,3}$ of an elastic medium are

$$
\rho(\boldsymbol{x}) \frac{\partial^{2} u_{j}}{\partial t^{2}}=\sum_{k=1}^{3} \frac{\partial \tau_{j k}}{\partial x_{k}}+S_{j}(t, \boldsymbol{x}), \quad j=1,2,3 .
$$

Here $\rho$ is the density of the medium and $\tau$ is the $3 \times 3$ stress tensor, which, in an isotropic medium is

$$
\tau_{j k}(t, \boldsymbol{x})=\lambda(\boldsymbol{x})\left[\sum_{l=1}^{3} \frac{\partial u_{l}(t, \boldsymbol{x})}{\partial x_{l}}\right] \mathbf{1}_{j}(k)+\mu(\boldsymbol{x})\left[\frac{\partial u_{j}(t, \boldsymbol{x})}{\partial x_{k}}+\frac{\partial u_{k}(t, \boldsymbol{x})}{\partial x_{j}}\right], \quad j, k=1,2,3,
$$

where $\lambda(\boldsymbol{x})$ and $\mu(\boldsymbol{x})$ are the Lamé parameters of the medium and $\mathbf{1}_{j}(k)=1$ if $k=j$ and 0 otherwise. The forcing term $\boldsymbol{S}(t, \boldsymbol{x})$ is the density of force sources.

In this paper we focus our attention on propagation through a random section occupying the region $x_{3} \in$ $(-L, 0)$ with the source $\boldsymbol{S}$ located outside of the random section at the surface $x_{3}=0$. The parameterization is motivated by waves probing for instance the heterogeneous earth and we may think of $x_{3}$ as the main probing direction. We shall refer to waves propagating in a direction with a negative (resp. positive) $x_{3^{-}}$ component as downward-moving (respectively upward-moving) waves.

It is more convenient to write the governing equations as a hyperbolic system. We introduce the new dependent variables, pressure $p$, three-dimensional velocity $\boldsymbol{\xi}$, and a stress tensor component $\boldsymbol{\chi}$, defined by

$$
\begin{aligned}
p(t, \boldsymbol{x}) & =\lambda(\boldsymbol{x})\left[\sum_{j=1}^{3} \frac{\partial u_{j}(t, \boldsymbol{x})}{\partial x_{j}}\right], \\
\xi_{j}(t, \boldsymbol{x}) & =\frac{\partial u_{j}(t, \boldsymbol{x})}{\partial t}, \quad j=1,2,3, \\
\chi_{j k}(t, \boldsymbol{x}) & =\mu(\boldsymbol{x})\left[\frac{\partial u_{j}(t, \boldsymbol{x})}{\partial x_{k}}+\frac{\partial u_{k}(t, \boldsymbol{x})}{\partial x_{j}}\right], \quad j, k=1,2,3 .
\end{aligned}
$$

Equations (1-2) can then be rewritten as

$$
\begin{aligned}
\rho(\boldsymbol{x}) \frac{\partial \xi_{j}}{\partial t} & =\frac{\partial p}{\partial x_{j}}+\sum_{k=1}^{3} \frac{\partial \chi_{j k}}{\partial x_{k}}+S_{j}(t, \boldsymbol{x}), \quad j=1,2,3 \\
\frac{\partial \chi_{j k}}{\partial t} & =\mu(\boldsymbol{x})\left[\frac{\partial \xi_{j}}{\partial x_{k}}+\frac{\partial \xi_{k}}{\partial x_{j}}\right], \quad j, k=1,2,3, \\
\frac{\partial p}{\partial t} & =\lambda(\boldsymbol{x})\left[\sum_{j=1}^{3} \frac{\partial \xi_{j}}{\partial x_{j}}\right] .
\end{aligned}
$$

If the shear modulus $\mu=0$, then $\chi=0$ and we get the acoustic wave equations for the pressure $p$ and the velocity $\boldsymbol{\xi}$.

We consider in this paper the situation in which a random section occupying the region $x_{3} \in(-L, 0)$ is sandwiched in between two homogeneous half-spaces. The medium parameters are: 


$$
\begin{gathered}
\frac{1}{\mu(\boldsymbol{x})}= \begin{cases}\mu_{0}^{-1} & \text { if } x_{3} \leq-L \text { or } x_{3} \geq 0, \\
\mu_{0}^{-1}\left[1+\varepsilon^{3} m_{\mu}\left(\frac{\boldsymbol{x}}{\varepsilon^{2}}\right)\right] & \text { if } x_{3} \in(-L, 0),\end{cases} \\
\rho(\boldsymbol{x})= \begin{cases}\rho_{0} & \text { if } x_{3} \leq-L \text { or } x_{3} \geq 0 \\
\rho_{0}\left[1+\varepsilon^{3} m_{\rho}\left(\frac{\boldsymbol{x}}{\varepsilon^{2}}\right)\right] & \text { if } x_{3} \in(-L, 0),\end{cases} \\
\lambda(\boldsymbol{x})= \begin{cases}\lambda_{0} & \text { if } x_{3} \leq-L \text { or } x_{3} \geq 0, \\
\lambda_{0}\left[1+\varepsilon^{3} m_{\lambda}\left(\frac{\boldsymbol{x}}{\varepsilon^{2}}\right)\right] & \text { if } x_{3} \in(-L, 0),\end{cases}
\end{gathered}
$$

with $\varepsilon$ a small parameter. The parameter $\varepsilon^{2}$ characterizes the ratio of the correlation length of the random medium fluctuations to the thickness of the random section. The random processes $m_{\mu}(\boldsymbol{x}), m_{\rho}(\boldsymbol{x})$, and $m_{\lambda}(\boldsymbol{x})$ describe the medium fluctuations. We assume that they are stationary and zero-mean and that they satisfy strong mixing conditions in $x_{3}$. We have also assume that the amplitude of the random fluctuations is of order $\varepsilon^{3}$. This is the interesting regime in which the medium fluctuations give rise to effective terms of order one when $\varepsilon$ goes to zero, as we will show in this paper.

Recall also that we let the source be located at the surface in the plane $x_{3}=0$. We consider a scaling regime in which the spatial support of the source (in the transverse direction), or equivalently the initial beam width, is of order $\varepsilon^{2}$. This means that the transverse scale of the source and the one of the spatial fluctuations of the medium are of the same order. Remember that the Rayleigh length for a beam with initial beam width $r_{0}$ and carrier wavelength $\lambda_{0}$ is of the order of $r_{0}^{2} / \lambda_{0}$ in absence of random fluctuations (the Rayleigh length is the distance from beam waist where the beam area is doubled by diffraction). In order to get a Rayleigh length of order one, we assume that the carrier wavelength of the source is of order $\varepsilon^{4}$. Therefore, in this regime the source has the form

$$
\boldsymbol{S}(t, \boldsymbol{x})=\boldsymbol{s}\left(\frac{t}{\varepsilon^{4}}, \frac{\boldsymbol{x}_{\perp}}{\varepsilon^{2}}\right) \delta\left(x_{3}\right), \quad \boldsymbol{x}=\left(\boldsymbol{x}_{\perp}, x_{3}\right), \quad \boldsymbol{x}_{\perp}=\left(x_{1}, x_{2}\right) .
$$

The source then generates waves that propagate mainly along the $x_{3}$-axis, as we will see below.

\section{The mode decomposition}

We rescale the transverse spatial variables so as to observe the wave at the scale of the source and we take a scaled Fourier transform in time:

$$
\check{\xi}_{j}^{\varepsilon}(\omega, \boldsymbol{x})=\int \xi_{j}\left(\varepsilon^{4} t, \varepsilon^{2} \boldsymbol{x}_{\perp}, x_{3}\right) e^{i \omega t} d t, \quad \check{\chi}_{j k}^{\varepsilon}(\omega, \boldsymbol{x})=\int \chi_{j k}\left(\varepsilon^{4} t, \varepsilon^{2} \boldsymbol{x}_{\perp}, x_{3}\right) e^{i \omega t} d t .
$$

Except at $x_{3}=0$ (where the source is) the six-dimensional vector $\left(\check{\xi}_{1}^{\varepsilon}, \check{\xi}_{2}^{\varepsilon}, \check{\xi}_{3}^{\varepsilon}, \check{\chi}_{13}^{\varepsilon}, \check{\chi}_{23}^{\varepsilon}, \check{\chi}_{33}^{\varepsilon}\right)$ satisfies

$$
\begin{aligned}
& \frac{\partial \check{\xi}_{1}^{\varepsilon}}{\partial x_{3}}=-\frac{i \omega}{\varepsilon^{4} \mu} \check{\chi}_{13}^{\varepsilon}-\frac{1}{\varepsilon^{2}} \frac{\partial \check{\xi}_{3}^{\varepsilon}}{\partial x_{1}}, \\
& \frac{\partial \check{\xi}_{2}^{\varepsilon}}{\partial x_{3}}=-\frac{i \omega}{\varepsilon^{4} \mu} \check{\chi}_{23}^{\varepsilon}-\frac{1}{\varepsilon^{2}} \frac{\partial \check{\xi}_{3}^{\varepsilon}}{\partial x_{2}}, \\
& \frac{\partial \check{\xi}_{3}^{\varepsilon}}{\partial x_{3}}=-\frac{i \omega}{2 \varepsilon^{4} \mu} \check{\chi}_{33}^{\varepsilon},
\end{aligned}
$$




$$
\begin{aligned}
\frac{\partial \check{\chi}_{13}^{\varepsilon}}{\partial x_{3}}= & -\frac{i \rho \omega}{\varepsilon^{4}} \check{\xi}_{1}^{\varepsilon}+\frac{\partial}{\partial x_{1}}\left(\frac{\lambda+2 \mu}{i \omega} \frac{\partial \check{\xi}_{1}^{\varepsilon}}{\partial x_{1}}\right)+\frac{\partial}{\partial x_{2}}\left(\frac{\mu}{i \omega} \frac{\partial \check{\xi}_{1}^{\varepsilon}}{\partial x_{2}}\right) \\
& +\frac{\partial}{\partial x_{1}}\left(\frac{\lambda}{i \omega} \frac{\partial \check{\xi}_{2}^{\varepsilon}}{\partial x_{2}}\right)+\frac{\partial}{\partial x_{2}}\left(\frac{\mu}{i \omega} \frac{\partial \check{\xi}_{2}^{\varepsilon}}{\partial x_{1}}\right)-\frac{1}{\varepsilon^{2}} \frac{\partial}{\partial x_{1}}\left(\frac{\lambda}{2 \mu} \check{\chi}_{33}^{\varepsilon}\right) \\
\frac{\partial \check{\chi}_{23}^{\varepsilon}}{\partial x_{3}}= & -\frac{i \rho \omega}{\varepsilon^{4}} \check{\xi}_{2}^{\varepsilon}+\frac{\partial}{\partial x_{2}}\left(\frac{\lambda+2 \mu}{i \omega} \frac{\partial \check{\xi}_{2}^{\varepsilon}}{\partial x_{2}}\right)+\frac{\partial}{\partial x_{1}}\left(\frac{\mu}{i \omega} \frac{\partial \check{\xi}_{2}^{\varepsilon}}{\partial x_{1}}\right) \\
& +\frac{\partial}{\partial x_{2}}\left(\frac{\lambda}{i \omega} \frac{\partial \check{\xi}_{1}^{\varepsilon}}{\partial x_{1}}\right)+\frac{\partial}{\partial x_{1}}\left(\frac{\mu}{i \omega} \frac{\partial \check{\xi}_{1}^{\varepsilon}}{\partial x_{2}}\right)-\frac{1}{\varepsilon^{2}} \frac{\partial}{\partial x_{2}}\left(\frac{\lambda}{2 \mu} \check{\chi}_{33}^{\varepsilon}\right) \\
\frac{\partial \check{\chi}_{33}^{\varepsilon}}{\partial x_{3}}= & \frac{2 \mu}{\lambda+2 \mu}\left[-\frac{i \rho \omega}{\varepsilon^{4}} \check{\xi}_{3}^{\varepsilon}-\frac{\lambda}{i \omega}\left(\frac{\partial^{2} \check{\xi}_{3}^{\varepsilon}}{\partial x_{1}^{2}}+\frac{\partial^{2} \check{\xi}_{3}^{\varepsilon}}{\partial x_{2}^{2}}\right)-\frac{1}{\varepsilon^{2}} \frac{\lambda+\mu}{\mu}\left(\frac{\partial \check{\chi}_{13}^{\varepsilon}}{\partial x_{1}}+\frac{\partial \check{\chi}_{23}^{\varepsilon}}{\partial x_{2}}\right)\right. \\
& \left.+\varepsilon^{2} \frac{\partial}{\partial x_{3}}\left(\frac{\lambda}{i \omega}\right)\left(\frac{\partial \check{\xi}_{1}^{\varepsilon}}{\partial x_{1}}+\frac{\partial \check{\xi}_{2}^{\varepsilon}}{\partial x_{2}}\right)-\frac{\partial}{\partial x_{3}}\left(\frac{\lambda}{2 \mu}\right) \check{\chi}_{33}^{\varepsilon}-\frac{\lambda}{\varepsilon^{2}} \frac{\partial}{\partial x_{1}}\left(\frac{1}{\mu}\right) \check{\chi}_{13}^{\varepsilon}-\frac{\lambda}{\varepsilon^{2}} \frac{\partial}{\partial x_{2}}\left(\frac{1}{\mu}\right) \check{\chi}_{23}^{\varepsilon}\right]
\end{aligned}
$$

where $\mu, \rho$ and $\lambda$ are evaluated at $\left(\varepsilon^{2} x_{1}, \varepsilon^{2} x_{2}, x_{3}\right)$. The four other dependent variables are given by:

$$
\begin{aligned}
\check{\chi}_{11}^{\varepsilon} & =-\frac{2 \varepsilon^{2} \mu}{i \omega} \frac{\partial \check{\xi}_{1}^{\varepsilon}}{\partial x_{1}}, \\
\check{\chi}_{12}^{\varepsilon} & =-\frac{\varepsilon^{2} \mu}{i \omega} \frac{\partial \check{\xi}_{1}^{\varepsilon}}{\partial x_{2}}-\frac{\varepsilon^{2} \mu}{i \omega} \frac{\partial \check{\xi}_{2}^{\varepsilon}}{\partial x_{1}}, \\
\check{\chi}_{22}^{\varepsilon} & =-\frac{2 \varepsilon^{2} \mu}{i \omega} \frac{\partial \check{\xi}_{2}^{\varepsilon}}{\partial x_{2}}, \\
\check{p} & =-\frac{\varepsilon^{2} \lambda}{i \omega} \frac{\partial \check{\xi}_{1}^{\varepsilon}}{\partial x_{1}}-\frac{\varepsilon^{2} \lambda}{i \omega} \frac{\partial \check{\xi}_{2}^{\varepsilon}}{\partial x_{2}}+\frac{\lambda}{2 \mu} \check{\chi}_{33}^{\varepsilon} .
\end{aligned}
$$

When the medium is homogeneous $\left(m_{\mu}=m_{\rho}=m_{\lambda}=0\right)$, then there exist plane wave solutions that depend only on $x_{3}$ and satisfy:

$$
\frac{d}{d x_{3}}\left(\begin{array}{c}
\check{\xi}_{1}^{\varepsilon} \\
\check{\chi}_{13}^{\varepsilon} \\
\check{\xi}_{2}^{\varepsilon} \\
\check{\chi}_{23}^{\varepsilon} \\
\check{\xi}_{3}^{\varepsilon} \\
\check{\chi}_{33}^{\varepsilon}
\end{array}\right)=-\frac{i \omega}{\varepsilon^{4}} \mathbf{M}\left(\begin{array}{c}
\check{\xi}_{1}^{\varepsilon} \\
\check{\chi}_{13}^{\varepsilon} \\
\check{\xi}_{2}^{\varepsilon} \\
\check{\chi}_{23}^{\varepsilon} \\
\check{\xi}_{3}^{\varepsilon} \\
\check{\chi}_{33}^{\varepsilon}
\end{array}\right), \quad \mathbf{M}=\left(\begin{array}{cccccc}
0 & \mu_{0}^{-1} & 0 & 0 & 0 & 0 \\
\rho_{0} & 0 & 0 & 0 & 0 & 0 \\
0 & 0 & 0 & \mu_{0}^{-1} & 0 & 0 \\
0 & 0 & \rho_{0} & 0 & 0 & 0 \\
0 & 0 & 0 & 0 & 0 & \left(2 \mu_{0}\right)^{-1} \\
0 & 0 & 0 & 0 & \frac{2 \mu_{0} \rho_{0}}{\lambda_{0}+2 \mu_{0}} & 0
\end{array}\right) .
$$

The diagonalization of the $6 \times 6$ matrix $\mathbf{M}$ gives the general form of the plane wave solutions

$$
\begin{aligned}
\check{\xi}_{1}^{\varepsilon}\left(\omega, x_{3}\right) & =\zeta_{S}^{-1 / 2}\left(\check{a}_{1}(\omega) e^{i \frac{\omega x_{3}}{c_{S} \varepsilon^{4}}}+\check{b}_{1}(\omega) e^{-i \frac{\omega x_{3}}{c_{S} \varepsilon^{4}}}\right), \\
\check{\chi}_{13}^{\varepsilon}\left(\omega, x_{3}\right) & =\zeta_{S}^{1 / 2}\left(-\check{a}_{1}(\omega) e^{i \frac{\omega x_{3}}{c_{S} \varepsilon^{4}}}+\check{b}_{1}(\omega) e^{-i \frac{\omega x_{3}}{c_{S} \varepsilon^{4}}}\right), \\
\check{\xi}_{2}^{\varepsilon}\left(\omega, x_{3}\right) & =\zeta_{S}^{-1 / 2}\left(\check{a}_{2}(\omega) e^{i \frac{\omega x_{3}}{c_{S} \varepsilon^{4}}}+\check{b}_{2}(\omega) e^{-i \frac{\omega x_{3}}{c_{S} \varepsilon^{4}}}\right), \\
\check{\chi}_{23}^{\varepsilon}\left(\omega, x_{3}\right) & =\zeta_{S}^{1 / 2}\left(-\check{a}_{2}(\omega) e^{i \frac{\omega x_{3}}{c_{S} \varepsilon^{4}}}+\check{b}_{2}(\omega) e^{-i \frac{\omega x_{3}}{c_{S} \varepsilon^{4}}}\right), \\
\check{\xi}_{3}^{\varepsilon}\left(\omega, x_{3}\right) & =\zeta_{P}^{-1 / 2}\left(\check{a}_{3}(\omega) e^{i \frac{\omega x_{3}}{c_{P} \varepsilon^{4}}}+\check{b}_{3}(\omega) e^{-i \frac{\omega x_{3}}{c_{P} \varepsilon^{4}}}\right), \\
\check{\chi}_{33}^{\varepsilon}\left(\omega, x_{3}\right) & =\zeta_{P}^{1 / 2}\left(-\check{a}_{3}(\omega) e^{i \frac{\omega x_{3}}{c_{P} \varepsilon^{4}}}+\check{b}_{3}(\omega) e^{-i \frac{\omega x_{3}}{c_{P} \varepsilon^{4}}}\right) .
\end{aligned}
$$

Here $c_{S}$ and $c_{P}$ are the shear-wave and pressure-wave velocities, and $\zeta_{S}$ and $\zeta_{P}$ are the shear-wave and pressure-wave impedances of the homogeneous medium:

$$
c_{S}=\sqrt{\frac{\mu_{0}}{\rho_{0}}}, \quad c_{P}=\sqrt{\frac{2 \mu_{0}+\lambda_{0}}{\rho_{0}}}, \quad \zeta_{S}=\sqrt{\mu_{0} \rho_{0}}, \quad \zeta_{P}=\sqrt{\frac{4 \mu_{0}^{2} \rho_{0}}{2 \mu_{0}+\lambda_{0}}} .
$$


The modes $\check{a}_{j}, j=1,2$ are upward-moving shear waves, the mode $\check{a}_{3}$ is an upward-moving pressure wave, the modes $\check{b}_{j}, j=1,2$ are downward-moving shear waves, and the mode $\breve{b}_{3}$ is a downward-moving pressure wave.

When the medium is heterogeneous and described by the model (9-11), then we introduce the generalized upward-moving modes $\check{a}_{j}^{\varepsilon}$ and downward-moving modes $\check{b}_{j}^{\varepsilon}$ defined by

$$
\begin{aligned}
\check{\xi}_{1}^{\varepsilon}(\omega, \boldsymbol{x}) & =\zeta_{S}^{-1 / 2}\left(\check{a}_{1}^{\varepsilon}(\omega, \boldsymbol{x}) e^{i \frac{\omega x_{3}}{c_{S} \varepsilon^{4}}}+\check{b}_{1}^{\varepsilon}(\omega, \boldsymbol{x}) e^{-i \frac{\omega x_{3}}{c_{S} \varepsilon^{4}}}\right), \\
\check{\chi}_{13}^{\varepsilon}(\omega, \boldsymbol{x}) & =\zeta_{S}^{1 / 2}\left(-\check{a}_{1}^{\varepsilon}(\omega, \boldsymbol{x}) e^{i \frac{\omega x_{3}}{c_{S} \varepsilon^{4}}}+\check{b}_{1}^{\varepsilon}(\omega, \boldsymbol{x}) e^{-i \frac{\omega x_{3}}{c_{S} \varepsilon^{4}}}\right), \\
\check{\xi}_{2}^{\varepsilon}(\omega, \boldsymbol{x}) & =\zeta_{S}^{-1 / 2}\left(\check{a}_{2}^{\varepsilon}(\omega, \boldsymbol{x}) e^{i \frac{\omega x_{3}}{c_{S} \varepsilon^{4}}}+\check{b}_{2}^{\varepsilon}(\omega, \boldsymbol{x}) e^{-i \frac{\omega x_{3}}{c_{S} \varepsilon^{4}}}\right), \\
\check{\chi}_{23}^{\varepsilon}(\omega, \boldsymbol{x}) & =\zeta_{S}^{1 / 2}\left(-\check{a}_{2}^{\varepsilon}(\omega, \boldsymbol{x}) e^{i \frac{\omega x_{3}}{c_{S} \varepsilon^{4}}}+\check{b}_{2}^{\varepsilon}(\omega, \boldsymbol{x}) e^{-i \frac{\omega x_{3}}{c_{S} \varepsilon^{4}}}\right), \\
\check{\xi}_{3}^{\varepsilon}(\omega, \boldsymbol{x}) & =\zeta_{P}^{-1 / 2}\left(\check{a}_{3}^{\varepsilon}(\omega, \boldsymbol{x}) e^{i \frac{\omega x_{3}}{c_{P} \varepsilon^{4}}}+\check{b}_{3}^{\varepsilon}(\omega, \boldsymbol{x}) e^{-i \frac{\omega x_{3}}{c_{P} \varepsilon^{4}}}\right), \\
\check{\chi}_{33}^{\varepsilon}(\omega, \boldsymbol{x}) & =\zeta_{P}^{1 / 2}\left(-\check{a}_{3}^{\varepsilon}(\omega, \boldsymbol{x}) e^{i \frac{\omega x_{3}}{c_{P} \varepsilon^{4}}}+\check{b}_{3}^{\varepsilon}(\omega, \boldsymbol{x}) e^{-i \frac{\omega x_{3}}{c_{P} \varepsilon^{4}}}\right),
\end{aligned}
$$

where $c_{S}, c_{P}, \zeta_{S}$, and $\zeta_{P}$ are the shear-wave and pressure-wave velocities and impedances of the background homogeneous medium as defined by $(29)$.

The radiation conditions at $+\infty$ and $-\infty$ derive from the fact that there is no downward-moving wave in the region $x_{3}>0$ and no upward-moving wave in the region $x_{3}<-L$ :

$$
\check{a}_{j}^{\varepsilon}\left(\omega, \boldsymbol{x}_{\perp}, x_{3}=(-L)^{-}\right)=0, \quad \check{b}_{j}^{\varepsilon}\left(\omega, \boldsymbol{x}_{\perp}, x_{3}=0^{+}\right)=0, \quad j=1,2,3,
$$

see Figure 1. The jump conditions across the source plane $x_{3}=0$ are obtained by integrating (6-8) across $x_{3}=0$ :

$$
\left[\check{\xi}_{j}^{\varepsilon}\right]_{0^{-}}^{0^{+}}=0, \quad\left[\check{\chi}_{13}^{\varepsilon}\right]_{0^{-}}^{0^{+}}=-\check{s}_{1}, \quad\left[\check{\chi}_{23}^{\varepsilon}\right]_{0^{-}}^{0^{+}}=-\check{s}_{2}, \quad\left[\check{\chi}_{33}^{\varepsilon}\right]_{0^{-}}^{0^{+}}=-\frac{2 \mu_{0}}{2 \mu_{0}+\lambda_{0}} \check{s}_{3}, \quad \check{\boldsymbol{s}}\left(\omega, \boldsymbol{x}_{\perp}\right)=\int \boldsymbol{s}\left(t, \boldsymbol{x}_{\perp}\right) e^{i \omega t} d t .
$$

From the jump conditions (37) and the radiation conditions at $+\infty$, we obtain:

$$
\begin{array}{cc}
\check{b}_{j}^{\varepsilon}\left(\omega, \boldsymbol{x}_{\perp}, x_{3}=0^{-}\right)=\check{b}_{\mathrm{inc}, j}\left(\omega, \boldsymbol{x}_{\perp}\right), \quad \check{b}_{\mathrm{inc}, j}\left(\omega, \boldsymbol{x}_{\perp}\right)=\frac{1}{2 \zeta_{S}^{1 / 2}} \check{s}_{j}\left(\omega, \boldsymbol{x}_{\perp}\right), \quad j=1,2, \\
\check{b}_{3}^{\varepsilon}\left(\omega, \boldsymbol{x}_{\perp}, x_{3}=0^{-}\right)=\check{b}_{\mathrm{inc}, 3}\left(\omega, \boldsymbol{x}_{\perp}\right), \quad & \check{b}_{\mathrm{inc}, 3}\left(\omega, \boldsymbol{x}_{\perp}\right)=\frac{1}{2 \zeta_{P}^{1 / 2}} \frac{2 \mu_{0}}{2 \mu_{0}+\lambda_{0}} \check{s}_{3}\left(\omega, \boldsymbol{x}_{\perp}\right) .
\end{array}
$$

By the continuity of the fields across $z=-L$ and the radiation condition at $-\infty$, we get:

$$
\check{a}_{j}^{\varepsilon}\left(\omega, \boldsymbol{x}_{\perp}, x_{3}=(-L)^{+}\right)=0, \quad j=1,2,3 .
$$

Let us next introduce the six-dimensional vector

$$
\check{\boldsymbol{X}}^{\varepsilon}\left(\omega, \boldsymbol{x}_{\perp}, x_{3}\right)=\left(\begin{array}{c}
\check{a}_{1}^{\varepsilon} \\
\check{b}_{1}^{\varepsilon} \\
\check{a}_{2}^{\varepsilon} \\
\check{b}_{2}^{\varepsilon} \\
\check{a}_{3}^{\varepsilon} \\
\check{b}_{3}^{\varepsilon}
\end{array}\right)\left(\omega, \boldsymbol{x}_{\perp}, x_{3}\right) .
$$

From (13-18), the vector $\check{\boldsymbol{X}}^{\varepsilon}$ satisfies in the section $x_{3} \in(-L, 0)$ the linear system 


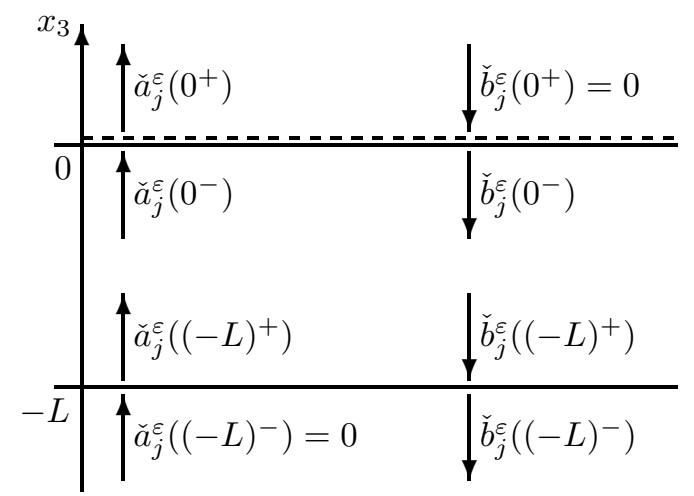

Fig. 1. Boundary conditions for the wave modes with a source at $x_{3}=0$ and radiation conditions at $\pm \infty$.

$$
\begin{aligned}
\frac{d \check{\boldsymbol{X}}^{\varepsilon}}{d x_{3}}= & \check{\mathbf{A}}^{\varepsilon}\left(\omega, \boldsymbol{x}_{\perp}, x_{3}\right) \check{\boldsymbol{X}}^{\varepsilon} \\
\check{\mathbf{A}}^{\varepsilon}\left(\omega, \boldsymbol{x}_{\perp}, x_{3}\right)= & \frac{1}{\varepsilon^{2}}\left[e^{i \frac{\omega x_{3}}{\varepsilon^{4}}\left(\frac{1}{c_{P}}+\frac{1}{c_{S}}\right)} \check{\mathbf{A}}^{1,1}+e^{i \frac{\omega x_{3}}{\varepsilon^{4}}\left(\frac{1}{c_{P}}-\frac{1}{c_{S}}\right)} \check{\mathbf{A}}^{1,-1}\right. \\
& \left.+e^{i \frac{\omega x_{3}}{\varepsilon^{4}}\left(-\frac{1}{c_{P}}+\frac{1}{c_{S}}\right)} \check{\mathbf{A}}^{-1,1}+e^{i \frac{\omega x_{3}}{\varepsilon^{4}}\left(-\frac{1}{c_{P}}-\frac{1}{c_{S}}\right)} \check{\mathbf{A}}^{-1,-1}\right] \\
& +\frac{1}{\varepsilon} \tilde{m}_{P}\left(\boldsymbol{x} \perp, \frac{x_{3}}{\varepsilon^{2}}\right)\left[e^{i \frac{2 \omega x_{3}}{\varepsilon^{4} c_{P}}} \check{\mathbf{B}}^{2,0}+e^{-i \frac{2 \omega x_{3}}{\varepsilon^{4} c_{P}}} \check{\mathbf{B}}^{-2,0}\right] \\
& +\frac{1}{\varepsilon} \tilde{m}_{S}\left(\boldsymbol{x}_{\perp}, \frac{x_{3}}{\varepsilon^{2}}\right)\left[e^{i \frac{2 \omega x_{3}}{\varepsilon^{4} c_{S}}} \check{\mathbf{B}}^{0,2}+e^{\left.-i \frac{2 \omega x_{3}}{\varepsilon^{4} c_{S}} \check{\mathbf{B}}^{0,-2}\right]}\right. \\
& +\frac{1}{\varepsilon} m_{P}\left(\boldsymbol{x}_{\perp}, \frac{x_{3}}{\varepsilon^{2}}\right) \check{\mathbf{B}}_{P}^{0,0}+\frac{1}{\varepsilon} m_{S}\left(\boldsymbol{x}_{\perp}, \frac{x_{3}}{\varepsilon^{2}}\right) \check{\mathbf{B}}_{S}^{0,0} \\
& +\check{\mathbf{A}}^{0,0}+e^{2 i \frac{\omega x_{3}}{\varepsilon^{4} c_{P}}} \check{\mathbf{A}}^{2,0}+e^{-2 i \frac{\omega x_{3}}{\varepsilon^{4} c_{P}}} \check{\mathbf{A}}^{-2,0}+e^{2 i \frac{\omega x_{3}}{\varepsilon^{4} c_{S}}} \check{\mathbf{A}}^{0,2}+e^{-2 i \frac{\omega x_{3}}{\varepsilon^{4} c_{S}}} \check{\mathbf{A}}^{0,-2}
\end{aligned}
$$

and the two-point boundary conditions

$$
\mathbf{H}^{-L} \check{\boldsymbol{X}}^{\varepsilon}\left(\omega, \boldsymbol{x}_{\perp},-L\right)+\mathbf{H}^{0} \check{\boldsymbol{X}}^{\varepsilon}\left(\omega, \boldsymbol{x}_{\perp}, 0\right)=\check{\boldsymbol{V}}\left(\omega, \boldsymbol{x}_{\perp}\right) .
$$

The matrices $\check{\mathbf{A}}^{j, k}$ and $\check{\mathbf{B}}^{j, k}$ are given in Appendix A. The vector $\check{\boldsymbol{V}}\left(\omega, \boldsymbol{x}_{\perp}\right)$ and the matrices $\mathbf{H}^{-L}$ and $\mathbf{H}^{0}$ are defined by

$$
\check{\boldsymbol{V}}\left(\omega, \boldsymbol{x}_{\perp}\right)=\left(\begin{array}{c}
0 \\
\check{b}_{\text {inc }, 1}\left(\omega, \boldsymbol{x}_{\perp}\right) \\
0 \\
\check{b}_{\text {inc }, 2}\left(\omega, \boldsymbol{x}_{\perp}\right) \\
0 \\
\check{b}_{\text {inc }, 3}\left(\omega, \boldsymbol{x}_{\perp}\right)
\end{array}\right), \quad \mathbf{H}^{-L}=\left(\begin{array}{cccccc}
1 & 0 & 0 & 0 & 0 & 0 \\
0 & 0 & 0 & 0 & 0 & 0 \\
0 & 0 & 1 & 0 & 0 & 0 \\
0 & 0 & 0 & 0 & 0 & 0 \\
0 & 0 & 0 & 0 & 1 & 0 \\
0 & 0 & 0 & 0 & 0 & 0
\end{array}\right), \quad \mathbf{H}^{0}=\left(\begin{array}{llllll}
0 & 0 & 0 & 0 & 0 & 0 \\
0 & 1 & 0 & 0 & 0 & 0 \\
0 & 0 & 0 & 0 & 0 & 0 \\
0 & 0 & 0 & 1 & 0 & 0 \\
0 & 0 & 0 & 0 & 0 & 0 \\
0 & 0 & 0 & 0 & 0 & 1
\end{array}\right),
$$

and

$$
\begin{aligned}
& m_{S}(\boldsymbol{x})=\left(m_{\mu}+m_{\rho}\right)(\boldsymbol{x}) \\
& m_{P}(\boldsymbol{x})=\left(\left(m_{\mu}+m_{\rho}\right)-\frac{\lambda_{0}}{2 \mu_{0}+\lambda_{0}}\left(m_{\mu}+m_{\lambda}\right)\right)(\boldsymbol{x}), \\
& \tilde{m}_{S}(\boldsymbol{x})=\left(m_{\mu}-m_{\rho}\right)(\boldsymbol{x}), \\
& \tilde{m}_{P}(\boldsymbol{x})=\left(\left(m_{\mu}-m_{\rho}\right)+\frac{\lambda_{0}}{2 \mu_{0}+\lambda_{0}}\left(m_{\mu}+m_{\lambda}\right)\right)(\boldsymbol{x}) .
\end{aligned}
$$

In the expression (43) of $\check{\mathbf{A}}^{\varepsilon}$ we have neglected terms of order $\varepsilon$ and smaller, and kept only the terms of order $\varepsilon^{-2}, \varepsilon^{-1}$ and 1. The mode decomposition (i.e. the definitions of the mode velocities and impedances) has canceled the terms of order $\varepsilon^{-4}$ that are present in the original equations (13-18). 
If the source is $\boldsymbol{x}_{\perp}$-independent and if the medium is homogeneous $\left(m_{\mu}=m_{\rho}=m_{\lambda}=0\right)$, then the solution of (42)-(44) is a constant vector which corresponds to a collection of three downward-moving modes: two shear-wave modes that propagate with the velocity $c_{S}$ and one pressure-wave mode that propagates with the velocity $c_{P}$

If the source is $\boldsymbol{x}_{\perp}$-dependent and/or the medium is heterogeneous, then transverse spatial effects and/or random effects have to be taken into account:

1) The matrices $\check{\mathbf{A}}^{j, k}$ in (43) correspond to deterministic transverse spatial effects. For $(j, k) \neq(0,0)$, these terms have large amplitudes, of order $\varepsilon^{-2}$, but they also have rapid phases that vary at the scale $\varepsilon^{4}$, and we will see that they give rise to effective terms of order one in the limit $\varepsilon \rightarrow 0$ through the application of an averaging theorem for highly oscillatory differential equations. These effects are purely deterministic, they consist of coupling between modes with different velocities, including coupling between upward- and downward-moving modes, and it is crucial to take them into account in the determination of the paraxial wave equation. Indeed, the matrix $\check{\mathbf{A}}^{0,0}$ that would be the natural candidate for the right-hand side of the paraxial wave equation does not capture the full paraxial mechanism. In other words, "brutally" suppressing the upward-moving (i.e. reflected) modes leads to wrong results. As we will see, the reflected modes are vanishing in the limit $\varepsilon \rightarrow 0$, but the coupling terms between upward- and downward-moving modes give important contributions to the dynamics of the transmitted waves.

2) The matrices $\check{\mathbf{B}}^{j, k}$ in (43) correspond to downward and upward scattering and coupling between modes due to the random heterogeneities of the medium. These terms have large amplitudes, of order $\varepsilon^{-1}$, but they vary rapidly at the scale $\varepsilon^{2}$, and the driving processes have mean zero and mixing properties. They will also give rise to effective terms of order one in the limit $\varepsilon \rightarrow 0$ through the application of a diffusion approximation theorem. The limit of $\check{\boldsymbol{X}}^{\varepsilon}$ will be characterized by a stochastic partial differential equation driven by two correlated Brownian fields.

\section{The paraxial wave equations}

In this section we state the main results of the paper. Proposition 1 describes the paraxial equations for elastic waves when the medium is homogeneous and it is proved in Section 5. Proposition 2 describes the white-noise paraxial equations for elastic waves when the medium is randomly heterogeneous and it is proved in Section 6.

Proposition 1 Let us assume that the medium is homogeneous $m_{\mu}=m_{\rho}=m_{\lambda}=0$. The transmitted wave consists of the succession of two fields that emerge at $x_{3}=-L$ around time $t=L / c_{P}$ and $t=L / c_{S}$ respectively:

$$
\begin{aligned}
& (\boldsymbol{\xi}, \boldsymbol{\chi}, p)\left(t=\frac{L}{c_{P}}+\varepsilon^{4} s, \varepsilon^{2} \boldsymbol{x}_{\perp}, x_{3}=-L\right) \stackrel{\varepsilon \rightarrow 0}{\longrightarrow}\left(\boldsymbol{\xi}_{P}, \chi_{P}, p_{P}\right)\left(s, \boldsymbol{x}_{\perp}\right), \\
& (\boldsymbol{\xi}, \boldsymbol{\chi}, p)\left(t=\frac{L}{c_{S}}+\varepsilon^{4} s, \varepsilon^{2} \boldsymbol{x}_{\perp}, x_{3}=-L\right) \stackrel{\varepsilon \rightarrow 0}{\longrightarrow}\left(\boldsymbol{\xi}_{S}, \boldsymbol{\chi}_{S}, p_{S}\right)\left(s, \boldsymbol{x}_{\perp}\right) .
\end{aligned}
$$

The first field $\left(\boldsymbol{\xi}_{P}, \chi_{P}, p_{P}\right)$ is a pressure wave of the form

$$
\begin{aligned}
\chi_{P j k}\left(s, \boldsymbol{x}_{\perp}\right) & =0, \quad(j, k) \neq(3,3), \\
\chi_{P 33}\left(s, \boldsymbol{x}_{\perp}\right) & =\frac{1}{2 \pi} \int \check{\chi}_{P 33}\left(\omega, \boldsymbol{x}_{\perp}\right) e^{-i \omega s} d \omega, \\
\xi_{P j}\left(s, \boldsymbol{x}_{\perp}\right) & =0, \quad j=1,2, \\
\xi_{P 3}\left(s, \boldsymbol{x}_{\perp}\right) & =\zeta_{P}^{-1} \chi_{P 33}\left(s, \boldsymbol{x}_{\perp}\right), \\
p_{P}\left(s, \boldsymbol{x}_{\perp}\right) & =\frac{\lambda_{0}}{2 \mu_{0}} \chi_{P 33}\left(s, \boldsymbol{x}_{\perp}\right) .
\end{aligned}
$$

The second field $\left(\boldsymbol{\xi}_{S}, \chi_{S}, p_{S}\right)$ is a shear wave of the form 


$$
\begin{aligned}
\chi_{S j 3}\left(s, \boldsymbol{x}_{\perp}\right) & =\frac{1}{2 \pi} \int \check{\chi}_{S j 3}\left(\omega, \boldsymbol{x}_{\perp}\right) e^{-i \omega s} d \omega, \quad j=1,2, \\
\chi_{S j k}\left(s, \boldsymbol{x}_{\perp}\right) & =0, \quad(j, k) \notin\{(1,3),(2,3)\}, \\
\xi_{S j}\left(s, \boldsymbol{x}_{\perp}\right) & =\zeta_{S}^{-1} \chi_{S j 3}\left(s, \boldsymbol{x}_{\perp}\right), \quad j=1,2, \\
\xi_{S 3}\left(s, \boldsymbol{x}_{\perp}\right) & =0, \\
p_{S}\left(s, \boldsymbol{x}_{\perp}\right) & =0 .
\end{aligned}
$$

The time-harmonic fields $\check{\chi}_{P 33}$ and $\check{\chi}_{S j 3}, j=1,2$, are given by

$$
\begin{aligned}
& \check{\chi}_{P 33}\left(\omega, \boldsymbol{x}_{\perp}\right)=\zeta_{P}^{1 / 2} \int \check{T}_{P}\left(\omega, \boldsymbol{x}_{\perp}-\boldsymbol{x}_{\perp}^{\prime}, 0\right) \check{b}_{\mathrm{inc}, 3}\left(\omega, \boldsymbol{x}_{\perp}^{\prime}\right) d \boldsymbol{x}_{\perp}^{\prime}, \\
& \check{\chi}_{S j 3}\left(\omega, \boldsymbol{x}_{\perp}\right)=\zeta_{S}^{1 / 2} \int \check{T}_{S}\left(\omega, \boldsymbol{x}_{\perp}-\boldsymbol{x}_{\perp}^{\prime}, 0\right) \check{b}_{\mathrm{inc}, j}\left(\omega, \boldsymbol{x}_{\perp}^{\prime}\right) d \boldsymbol{x}_{\perp}^{\prime}, \quad j=1,2,
\end{aligned}
$$

where $\check{T}_{P}\left(\omega, \boldsymbol{x}_{\perp}, x_{3}\right)$ and $\check{T}_{S}\left(\omega, \boldsymbol{x}_{\perp}, x_{3}\right)$ satisfy the Schrödinger equations for $x_{3} \in(-L, 0)$ :

$$
\begin{array}{ll}
\frac{\partial \check{T}_{P}\left(\omega, \boldsymbol{x}_{\perp}, x_{3}\right)}{\partial x_{3}}=\frac{i c_{P}}{2 \omega} \Delta_{\boldsymbol{x}_{\perp}} \check{T}_{P}\left(\omega, \boldsymbol{x}_{\perp}, x_{3}\right), & \check{T}_{P}\left(\omega, \boldsymbol{x}_{\perp}, x_{3}=-L\right)=\delta\left(\boldsymbol{x}_{\perp}\right), \\
\frac{\partial \check{T}_{S}\left(\omega, \boldsymbol{x}_{\perp}, x_{3}\right)}{\partial x_{3}}=\frac{i c_{S}}{2 \omega} \Delta_{\boldsymbol{x}_{\perp}} \check{T}_{S}\left(\omega, \boldsymbol{x}_{\perp}, x_{3}\right), & \check{T}_{S}\left(\omega, \boldsymbol{x}_{\perp}, x_{3}=-L\right)=\delta\left(\boldsymbol{x}_{\perp}\right) .
\end{array}
$$

Here $\Delta_{\boldsymbol{x}_{\perp}}=\frac{\partial^{2}}{\partial x_{1}^{2}}+\frac{\partial^{2}}{\partial x_{2}^{2}}$ is the transverse Laplacian.

We now give the result when the medium has random fluctuations.

Proposition 2 In the limit $\varepsilon \rightarrow 0$, the transmitted wave consists of the succession of two fields that emerge at $x_{3}=-L$ around time $t=L / c_{P}$ and $t=L / c_{S}$ as described by (50-51). The first field is a pressure wave of the form (52-56). The second field is a shear wave of the form (57-61). The time-harmonic fields $\check{\chi}_{P 33}$ and $\check{\chi}_{S j 3}, j=1,2$ are given by

$$
\begin{aligned}
& \check{\chi}_{P 33}\left(\omega, \boldsymbol{x}_{\perp}\right)=\zeta_{P}^{1 / 2} \int \check{\mathcal{T}}_{P}\left(\omega, \boldsymbol{x}_{\perp}, \boldsymbol{x}_{\perp}^{\prime}, 0\right) \check{b}_{\mathrm{inc}, 3}\left(\omega, \boldsymbol{x}_{\perp}^{\prime}\right) d \boldsymbol{x}_{\perp}^{\prime}, \\
& \check{\chi}_{S j 3}\left(\omega, \boldsymbol{x}_{\perp}\right)=\zeta_{S}^{1 / 2} \int \check{\mathcal{T}}_{S}\left(\omega, \boldsymbol{x}_{\perp}, \boldsymbol{x}_{\perp}^{\prime}, 0\right) \check{b}_{\mathrm{inc}, j}\left(\omega, \boldsymbol{x}_{\perp}^{\prime}\right) d \boldsymbol{x}_{\perp}^{\prime}, \quad j=1,2 .
\end{aligned}
$$

The operators $\check{\mathcal{T}}_{P}$ and $\check{\mathcal{T}}_{S}$ are the solutions of the following Itô-Schrödinger diffusion models for $x_{3} \in(-L, 0)$ :

$$
\begin{aligned}
& d \check{\mathcal{T}}_{P}\left(\omega, \boldsymbol{x}_{\perp}, \boldsymbol{x}_{\perp}^{\prime}, x_{3}\right)=\frac{i c_{P}}{2 \omega} \Delta_{\boldsymbol{x}_{\perp}^{\prime}} \check{\mathcal{T}}_{P}\left(\omega, \boldsymbol{x}_{\perp}, \boldsymbol{x}_{\perp}^{\prime}, x_{3}\right) d x_{3}+\frac{i \omega}{2 c_{P}} \check{\mathcal{T}}_{P}\left(\omega, \boldsymbol{x}_{\perp}, \boldsymbol{x}_{\perp}^{\prime}, x_{3}\right) \circ d B_{P}\left(\boldsymbol{x}_{\perp}^{\prime}, x_{3}\right), \\
& d \check{\mathcal{T}}_{S}\left(\omega, \boldsymbol{x}_{\perp}, \boldsymbol{x}_{\perp}^{\prime}, x_{3}\right)=\frac{i c_{S}}{2 \omega} \Delta_{\boldsymbol{x}_{\perp}^{\prime}} \check{\mathcal{T}}_{S}\left(\omega, \boldsymbol{x}_{\perp}, \boldsymbol{x}_{\perp}^{\prime}, x_{3}\right) d x_{3}+\frac{i \omega}{2 c_{S}} \check{\mathcal{T}}_{S}\left(\omega, \boldsymbol{x}_{\perp}, \boldsymbol{x}_{\perp}^{\prime}, x_{3}\right) \circ d B_{S}\left(\boldsymbol{x}_{\perp}^{\prime}, x_{3}\right),
\end{aligned}
$$

with the initial conditions

$$
\check{\mathcal{T}}_{P}\left(\omega, \boldsymbol{x}_{\perp}, \boldsymbol{x}_{\perp}^{\prime}, x_{3}=-L\right)=\check{\mathcal{T}}_{S}\left(\omega, \boldsymbol{x}_{\perp}, \boldsymbol{x}_{\perp}^{\prime}, x_{3}=-L\right)=\delta\left(\boldsymbol{x}_{\perp}-\boldsymbol{x}_{\perp}^{\prime}\right) .
$$

Here the symbol $\circ$ stands for the Stratonovich stochastic integral, $B_{P}\left(\boldsymbol{x}_{\perp}, x_{3}\right)$ and $B_{S}\left(\boldsymbol{x}_{\perp}, x_{3}\right)$ are two correlated Brownian fields with the covariance matrix

$$
\begin{aligned}
& \left(\begin{array}{l}
\mathbb{E}\left[B_{P}\left(\boldsymbol{x}_{\perp}, x_{3}\right) B_{P}\left(, \boldsymbol{x}_{\perp}^{\prime}, x_{3}^{\prime}\right)\right] \mathbb{E}\left[B_{P}\left(\boldsymbol{x}_{\perp}, x_{3}\right) B_{S}\left(, \boldsymbol{x}_{\perp}^{\prime}, x_{3}^{\prime}\right)\right] \\
\mathbb{E}\left[B_{S}\left(\boldsymbol{x}_{\perp}, x_{3}\right) B_{P}\left(, \boldsymbol{x}_{\perp}^{\prime}, x_{3}^{\prime}\right)\right] \mathbb{E}\left[B_{S}\left(\boldsymbol{x}_{\perp}, x_{3}\right) B_{S}\left(, \boldsymbol{x}_{\perp}^{\prime}, x_{3}^{\prime}\right)\right]
\end{array}\right)=\min \left\{x_{3}, x_{3}^{\prime}\right\} \mathbf{C}_{0}\left(\boldsymbol{x}_{\perp}-\boldsymbol{x}_{\perp}^{\prime}\right), \\
& \mathbf{C}_{0}\left(\boldsymbol{x}_{\perp}\right)=\left(\begin{array}{ll}
C_{0, P P}\left(\boldsymbol{x}_{\perp}\right) & C_{0, P S}\left(\boldsymbol{x}_{\perp}\right) \\
C_{0, P S}\left(\boldsymbol{x}_{\perp}\right) & C_{0, S S}\left(\boldsymbol{x}_{\perp}\right)
\end{array}\right),
\end{aligned}
$$

and we have used the notations 


$$
\begin{aligned}
C_{P P}(\boldsymbol{x}) & =\mathbb{E}\left[m_{P}\left(\boldsymbol{x}^{\prime}+\boldsymbol{x}\right) m_{P}\left(\boldsymbol{x}^{\prime}\right)\right], \\
C_{0, P P}\left(\boldsymbol{x}_{\perp}\right) & =\int_{-\infty}^{\infty} C_{P P}\left(\boldsymbol{x}_{\perp}, x_{3}\right) d x_{3}, \\
C_{S S}(\boldsymbol{x}) & =\mathbb{E}\left[m_{S}\left(\boldsymbol{x}^{\prime}+\boldsymbol{x}\right) m_{S}\left(\boldsymbol{x}^{\prime}\right)\right], \\
C_{0, S S}\left(\boldsymbol{x}_{\perp}\right) & =\int_{-\infty}^{\infty} C_{S S}\left(\boldsymbol{x}_{\perp}, x_{3}\right) d x_{3}, \\
C_{P S}(\boldsymbol{x}) & =\frac{1}{2} \mathbb{E}\left[m_{P}\left(\boldsymbol{x}^{\prime}+\boldsymbol{x}\right) m_{S}\left(\boldsymbol{x}^{\prime}\right)\right]+\frac{1}{2} \mathbb{E}\left[m_{S}\left(\boldsymbol{x}^{\prime}+\boldsymbol{x}\right) m_{P}\left(\boldsymbol{x}^{\prime}\right)\right], \\
C_{0, P S}\left(\boldsymbol{x}_{\perp}\right) & =\int_{-\infty}^{\infty} C_{P S}\left(\boldsymbol{x}_{\perp}, x_{3}\right) d x_{3},
\end{aligned}
$$

where $m_{P}$ and $m_{S}$ are defined by (46-47).

In order to give a complete characterization of the transmitted field, we add that there is no other transmitted wave in the sense that for any time $t_{0} \notin\left\{L / c_{P}, L / c_{S}\right\}$,

$$
(\boldsymbol{\xi}, \boldsymbol{\chi}, p)\left(t=t_{0}+\varepsilon^{4} s, \varepsilon^{2} \boldsymbol{x}_{\perp}, x_{3}=-L\right) \stackrel{\varepsilon \rightarrow 0}{\longrightarrow}(\mathbf{0}, \mathbf{0}, 0) .
$$

There is no reflected wave in the sense that for any time $t_{0}>0$,

$$
(\boldsymbol{\xi}, \boldsymbol{\chi}, p)\left(t=t_{0}+\varepsilon^{4} s, \varepsilon^{2} \boldsymbol{x}_{\perp}, x_{3}=0\right) \stackrel{\varepsilon \rightarrow 0}{\longrightarrow}(\mathbf{0}, \mathbf{0}, 0) .
$$

In [7] the existence and uniqueness has been established for each of the two random processes

$$
\begin{aligned}
& V_{P}\left(\omega, \boldsymbol{x}_{\perp}, x_{3}\right)=\int \check{\mathcal{T}}_{P}\left(\omega, \boldsymbol{x}_{\perp}, \boldsymbol{x}_{\perp}^{\prime}, x_{3}\right) \phi_{P}\left(\boldsymbol{x}_{\perp}^{\prime}\right) d \boldsymbol{x}_{\perp}^{\prime}, \\
& V_{S}\left(\omega, \boldsymbol{x}_{\perp}, x_{3}\right)=\int \check{\mathcal{T}}_{S}\left(\omega, \boldsymbol{x}_{\perp}, \boldsymbol{x}_{\perp}^{\prime}, x_{3}\right) \phi_{S}\left(\boldsymbol{x}_{\perp}^{\prime}\right) d \boldsymbol{x}_{\perp}^{\prime},
\end{aligned}
$$

for any test functions $\phi_{S}$ and $\phi_{P}$ with unit $L^{2}\left(\mathbb{R}^{2}, \mathbb{C}\right)$-norm. Furthermore, it is shown that each of the two processes $V_{S}\left(\omega, \boldsymbol{x}_{\perp}, x_{3}\right)$ and $V_{P}\left(\omega, \boldsymbol{x}_{\perp}, x_{3}\right)$ is a continuous Markov diffusion process on the unit ball of $L^{2}\left(\mathbb{R}^{2}, \mathbb{C}\right)$. The moment equations moreover satisfy a closed system at each order [12]. It is straightforward to generalize these results for the joint distribution of the pair of processes $\left(V_{S}, V_{P}\right)$. The interested reader can find explicit calculations for the mean intensity, the autocorrelation function, and other relevant quantities of the transmitted wave in [14].

The fact that the solutions of Itô-Schrödinger equations have constant $L^{2}$-norms shows that the sum of the energies of the transmitted pressure wave (50) and of the transmitted shear wave (51) (in homogeneous or random media) is equal to the energy of the incoming wave. Indeed, the energy flux density is given by

$$
\mathcal{F}(t, \boldsymbol{x})=\left[\lambda(\nabla \cdot \boldsymbol{u}) \mathbf{I}+\mu\left(\nabla \boldsymbol{u}+\nabla^{t} \boldsymbol{u}\right)\right] \boldsymbol{\xi}=p \boldsymbol{\xi}+\chi \boldsymbol{\xi} .
$$

Therefore, the total energy flux generated by the source and entering the random section at $x_{3}=0$ is

$$
\mathcal{F}_{\text {inc }}=\frac{1}{8 \pi \rho_{0}} \iint \frac{\left|\check{s}_{1}\left(\omega, \boldsymbol{x}_{\perp}\right)\right|^{2}}{c_{S}}+\frac{\left|\check{s}_{2}\left(\omega, \boldsymbol{x}_{\perp}\right)\right|^{2}}{c_{S}}+\frac{\left|\check{s}_{3}\left(\omega, \boldsymbol{x}_{\perp}\right)\right|^{2}}{c_{P}} d \omega d \boldsymbol{x}_{\perp}
$$

the energy flux of the transmitted pressure wave (50) exiting at $x_{3}=-L$ is

$$
\mathcal{F}_{\mathrm{tr}, P}=\frac{1}{8 \pi \rho_{0}} \iint \frac{1}{c_{P}}\left|\int \check{\mathcal{T}}_{P}\left(\omega, \boldsymbol{x}_{\perp}, \boldsymbol{x}_{\perp}^{\prime}, 0\right) \check{s}_{3}\left(\omega, \boldsymbol{x}_{\perp}^{\prime}\right) d \boldsymbol{x}_{\perp}^{\prime}\right|^{2} d \omega d \boldsymbol{x}_{\perp}=\frac{1}{8 \pi \rho_{0}} \iint \frac{\left|\check{s}_{3}\left(\omega, \boldsymbol{x}_{\perp}\right)\right|^{2}}{c_{P}} d \omega d \boldsymbol{x}_{\perp},
$$

and the energy flux of the transmitted shear wave (51) exiting at $x_{3}=-L$ is

$$
\mathcal{F}_{\mathrm{tr}, S}=\frac{1}{8 \pi \rho_{0}} \sum_{j=1}^{2} \iint \frac{1}{c_{S}}\left|\int \check{\mathcal{T}}_{S}\left(\omega, \boldsymbol{x}_{\perp}, \boldsymbol{x}_{\perp}^{\prime}, 0\right) \check{s}_{j}\left(\omega, \boldsymbol{x}_{\perp}^{\prime}\right) d \boldsymbol{x}_{\perp}^{\prime}\right|^{2} d \omega d \boldsymbol{x}_{\perp}=\frac{1}{8 \pi \rho_{0}} \sum_{j=1}^{2} \iint \frac{\left|\check{s}_{j}\left(\omega, \boldsymbol{x}_{\perp}\right)\right|^{2}}{c_{S}} d \omega d \boldsymbol{x}_{\perp} .
$$

This shows that we have $\mathcal{F}_{\text {inc }}=\mathcal{F}_{\mathrm{tr}, P}+\mathcal{F}_{\mathrm{tr}, S}$, which illustrates the fact that no energy is reflected and all the energy is transmitted in the form of the two wave fields described in the propositions. 


\section{The derivation of the paraxial wave equations in the homogeneous case}

In this section we assume that there are no random fluctuations $m_{\mu}=m_{\rho}=m_{\lambda}=0$ and we prove Proposition 1. We transform the two-point boundary value problem (42)-(44) into an initial value problem. This is done by an invariant imbedding step in which we introduce transmission and reflection matrices. First, we define the lateral Fourier modes for $j=1,2,3$ :

$$
\widehat{a}_{j}^{\varepsilon}\left(\omega, \boldsymbol{\kappa}_{\perp}, x_{3}\right)=\int \check{a}_{j}^{\varepsilon}\left(\omega, \boldsymbol{x}_{\perp}, x_{3}\right) e^{-i \boldsymbol{\kappa}_{\perp} \cdot \boldsymbol{x}_{\perp}} d \boldsymbol{x}_{\perp}, \quad \widehat{b}_{j}^{\varepsilon}\left(\omega, \boldsymbol{\kappa}_{\perp}, x_{3}\right)=\int \check{b}_{j}^{\varepsilon}\left(\omega, \boldsymbol{x}_{\perp}, x_{3}\right) e^{-i \boldsymbol{\kappa}_{\perp} \cdot \boldsymbol{x}_{\perp}} d \boldsymbol{x}_{\perp},
$$

where we have denoted $\boldsymbol{\kappa}_{\perp}=\left(\kappa_{1}, \kappa_{2}\right)$. The reflected wave is characterized by $\widehat{a}_{j}^{\varepsilon}\left(\omega, \boldsymbol{\kappa}_{\perp}, x_{3}=0\right)$ and the transmitted wave by $\widehat{b}_{j}^{\varepsilon}\left(\omega, \boldsymbol{\kappa}_{\perp}, x_{3}=-L\right)$. The parameters $\omega$ and $\boldsymbol{\kappa}_{\perp}$ are frozen in the problem, so we shall not write explicitly the $\left(\omega, \boldsymbol{\kappa}_{\perp}\right)$-dependence of the vectors and matrices. The vector $\widehat{\boldsymbol{X}}^{\varepsilon}\left(x_{3}\right)$ is defined as in (41), however, in terms of the Fourier modes $\widehat{a}_{j}^{\varepsilon}$ and $\widehat{b}_{j}^{\varepsilon}$ and it is the solution of the two-point boundary value problem:

$$
\frac{d \widehat{\boldsymbol{X}}^{\varepsilon}}{d x_{3}}=\widehat{\mathbf{A}}^{\varepsilon}\left(x_{3}\right) \widehat{\boldsymbol{X}}^{\varepsilon}, \quad \mathbf{H}^{-L} \widehat{\boldsymbol{X}}^{\varepsilon}(-L)+\mathbf{H}^{0} \widehat{\boldsymbol{X}}^{\varepsilon}(0)=\widehat{\boldsymbol{V}},
$$

with the $6 \times 6$ matrix $\widehat{\mathbf{A}}^{\varepsilon}\left(x_{3}\right)$ given by

$$
\begin{aligned}
\widehat{\mathbf{A}}^{\varepsilon}\left(x_{3}\right)= & \frac{1}{\varepsilon^{2}}\left[e^{i \frac{\omega x_{3}}{\varepsilon^{4}}\left(\frac{1}{c_{P}}+\frac{1}{c_{S}}\right)} \widehat{\mathbf{A}}^{1,1}+e^{i \frac{\omega x_{3}}{\varepsilon^{4}}\left(\frac{1}{c_{P}}-\frac{1}{c_{S}}\right)} \widehat{\mathbf{A}}^{1,-1}+e^{i \frac{\omega x_{3}}{\varepsilon^{4}}\left(-\frac{1}{c_{P}}+\frac{1}{c_{S}}\right)} \widehat{\mathbf{A}}^{-1,1}+e^{i \frac{\omega x_{3}}{\varepsilon^{4}}\left(-\frac{1}{c_{P}}-\frac{1}{c_{S}}\right)} \widehat{\mathbf{A}}^{-1,-1}\right] \\
& +\widehat{\mathbf{A}}^{0,0}+e^{2 i \frac{\omega x_{3}}{\varepsilon^{4} c_{P}}} \check{\mathbf{A}}^{2,0}+e^{-2 i \frac{\omega x_{3}}{\varepsilon^{4} c_{P}}} \check{\mathbf{A}}^{-2,0}+e^{2 i \frac{\omega x_{3}}{\varepsilon^{4} c_{S}}} \check{\mathbf{A}}^{0,2}+e^{-2 i \frac{\omega x_{3}}{\varepsilon^{4} c_{S}}} \check{\mathbf{A}}^{0,-2},
\end{aligned}
$$

$\widehat{\mathbf{A}}^{j, k}$ are given in Appendix A, $\mathbf{H}^{-L}$ and $\mathbf{H}^{0}$ are given by (45), and

$$
\widehat{\boldsymbol{V}}=\left(\begin{array}{c}
0 \\
\widehat{b}_{\mathrm{inc}, 1} \\
0 \\
\widehat{b}_{\mathrm{inc}, 2} \\
0 \\
\widehat{b}_{\mathrm{inc}, 3}
\end{array}\right), \quad \widehat{b}_{\mathrm{inc}, j}\left(\omega, \boldsymbol{\kappa}_{\perp}\right)=\int \check{b}_{\mathrm{inc}, j}\left(\omega, \boldsymbol{x}_{\perp}\right) e^{-i \boldsymbol{\kappa}_{\perp} \cdot \boldsymbol{x}_{\perp}} d \boldsymbol{x}_{\perp}, \quad j=1,2,3 .
$$

By applying Proposition B.1 of Appendix B, we get that the reflected and transmitted modes are given by

$$
\begin{array}{ll}
\widehat{a}_{j}^{\varepsilon}\left(x_{3}=0\right)=\left[\widehat{\mathbf{R}}^{\varepsilon}\left(x_{3}=0\right) \widehat{\boldsymbol{V}}\right]_{2 j-1}, & j=1,2,3, \\
\widehat{b}_{j}^{\varepsilon}\left(x_{3}=-L\right)=\left[\widehat{\mathbf{T}}^{\varepsilon}\left(x_{3}=0\right) \widehat{\boldsymbol{V}}\right]_{2 j}, & j=1,2,3,
\end{array}
$$

where the reflection and transmission matrices $\widehat{\mathbf{R}}^{\varepsilon}$ and $\widehat{\mathbf{T}}^{\varepsilon}$ are solution of the initial value problem

$$
\begin{aligned}
& \frac{d \widehat{\mathbf{R}}^{\varepsilon}}{d x_{3}}=\left(\mathbf{I}-\widehat{\mathbf{R}}^{\varepsilon} \mathbf{H}^{0}\right) \widehat{\mathbf{A}}^{\varepsilon}\left(x_{3}\right) \widehat{\mathbf{R}}^{\varepsilon}, \quad \widehat{\mathbf{R}}^{\varepsilon}\left(x_{3}=-L\right)=\mathbf{I}, \\
& \frac{d \widehat{\mathbf{T}}^{\varepsilon}}{d x_{3}}=-\widehat{\mathbf{T}}^{\varepsilon} \mathbf{H}^{0} \widehat{\mathbf{A}}^{\varepsilon}\left(x_{3}\right) \widehat{\mathbf{R}}^{\varepsilon}, \quad \widehat{\mathbf{T}}^{\varepsilon}\left(x_{3}=-L\right)=\mathbf{I},
\end{aligned}
$$

and $\mathbf{I}$ is the $6 \times 6$ identity matrix. Note that the linear boundary value problem has been transformed into a nonlinear initial value problem, that has the form of a matrix Riccati equation. We can now apply Proposition C.1 which gives the asymptotic behavior of the reflection and transmission matrices: 


$$
\begin{aligned}
\frac{d \widehat{\mathbf{R}}}{d x_{3}}= & \left(\mathbf{I}-\widehat{\mathbf{R}} \mathbf{H}^{0}\right) \widehat{\mathbf{A}}^{0,0} \widehat{\mathbf{R}}+\frac{i}{\omega\left(\frac{1}{c_{P}}+\frac{1}{c_{S}}\right)}\left[\left(\mathbf{I}-\widehat{\mathbf{R}} \mathbf{H}^{0}\right) \widehat{\mathbf{A}}^{1,1} \widehat{\mathbf{R}},\left(\mathbf{I}-\widehat{\mathbf{R}} \mathbf{H}^{0}\right) \widehat{\mathbf{A}}^{-1,-1} \widehat{\mathbf{R}}\right] \\
& +\frac{i}{\omega\left(\frac{1}{c_{P}}-\frac{1}{c_{S}}\right)}\left[\left(\mathbf{I}-\widehat{\mathbf{R}} \mathbf{H}^{0}\right) \widehat{\mathbf{A}}^{1,-1} \widehat{\mathbf{R}},\left(\mathbf{I}-\widehat{\mathbf{R}} \mathbf{H}^{0}\right) \widehat{\mathbf{A}}^{-1,1} \widehat{\mathbf{R}}\right], \\
\frac{d \widehat{\mathbf{T}}}{d x_{3}}= & -\widehat{\mathbf{T}} \mathbf{H}^{0} \widehat{\mathbf{A}}^{0,0} \widehat{\mathbf{R}}+\frac{i}{\omega\left(\frac{1}{c_{P}}+\frac{1}{c_{S}}\right)}\left[\widehat{\mathbf{T}} \mathbf{H}^{0} \widehat{\mathbf{A}}{ }^{1,1} \widehat{\mathbf{R}}, \widehat{\mathbf{T}} \mathbf{H}^{0} \widehat{\mathbf{A}}^{-1,-1} \widehat{\mathbf{R}}\right] \\
& +\frac{i}{\omega\left(\frac{1}{c_{P}}-\frac{1}{c_{S}}\right)}\left[\widehat{\mathbf{T}} \mathbf{H}^{0} \widehat{\mathbf{A}}^{1,-1} \widehat{\mathbf{R}}, \widehat{\mathbf{T}} \mathbf{H}^{0} \widehat{\mathbf{A}}^{-1,1} \widehat{\mathbf{R}}\right],
\end{aligned}
$$

where the brackets stand for Lie brackets as defined in Proposition C.1. It turns out that these equations can be dramatically simplified. Indeed, the Lie brackets can be computed

$$
\begin{aligned}
{\left[\left(\mathbf{I}-\widehat{\mathbf{R}} \mathbf{H}^{0}\right) \widehat{\mathbf{A}}^{1,1} \widehat{\mathbf{R}},\left(\mathbf{I}-\widehat{\mathbf{R}} \mathbf{H}^{0}\right) \widehat{\mathbf{A}}^{-1,-1} \widehat{\mathbf{R}}\right] } & =\left(\mathbf{I}-\widehat{\mathbf{R}} \mathbf{H}^{0}\right)\left(\widehat{\mathbf{A}}^{1,1} \widehat{\mathbf{A}}^{-1,-1}-\widehat{\mathbf{A}}^{-1,-1} \widehat{\mathbf{A}}^{1,1}\right) \widehat{\mathbf{R}}, \\
{\left[\left(\mathbf{I}-\widehat{\mathbf{R}} \mathbf{H}^{0}\right) \widehat{\mathbf{A}}^{1,-1} \widehat{\mathbf{R}},\left(\mathbf{I}-\widehat{\mathbf{R}} \mathbf{H}^{0}\right) \widehat{\mathbf{A}}^{-1,1} \widehat{\mathbf{R}}\right] } & =\left(\mathbf{I}-\widehat{\mathbf{R}} \mathbf{H}^{0}\right)\left(\widehat{\mathbf{A}}^{1,-1} \widehat{\mathbf{A}}^{-1,1}-\widehat{\mathbf{A}}^{-1,1} \widehat{\mathbf{A}}^{1,-1}\right) \widehat{\mathbf{R}}, \\
{\left[\widehat{\mathbf{T}} \mathbf{H}^{0} \widehat{\mathbf{A}}{ }^{1,1} \widehat{\mathbf{R}}, \widehat{\mathbf{T}} \mathbf{H}^{0} \widehat{\mathbf{A}}^{-1,-1} \widehat{\mathbf{R}}\right] } & =\widehat{\mathbf{T}}\left(\widehat{\mathbf{A}}^{1,1} \widehat{\mathbf{A}}^{-1,-1}-\widehat{\mathbf{A}}^{-1,-1} \widehat{\mathbf{A}}^{1,1}\right) \mathbf{H}^{0} \widehat{\mathbf{R}}, \\
{\left[\widehat{\mathbf{T}} \mathbf{H}^{0} \widehat{\mathbf{A}}^{1,-1} \widehat{\mathbf{R}}, \widehat{\mathbf{T}} \mathbf{H}^{0} \widehat{\mathbf{A}}^{-1,1} \widehat{\mathbf{R}}\right] } & =\widehat{\mathbf{T}}\left(\widehat{\mathbf{A}}^{1,-1} \widehat{\mathbf{A}}^{-1,1}-\widehat{\mathbf{A}}^{-1,1} \widehat{\mathbf{A}}^{1,-1}\right) \mathbf{H}^{0} \widehat{\mathbf{R}},
\end{aligned}
$$

and we have the remarkable identity

$$
\widehat{\mathbf{A}}^{0,0}+\frac{i}{\omega\left(\frac{1}{c_{P}}+\frac{1}{c_{S}}\right)}\left(\widehat{\mathbf{A}}^{1,1} \widehat{\mathbf{A}}^{-1,-1}-\widehat{\mathbf{A}}^{-1,-1} \widehat{\mathbf{A}}^{1,1}\right)+\frac{i}{\omega\left(\frac{1}{c_{P}}-\frac{1}{c_{S}}\right)}\left(\widehat{\mathbf{A}}^{1,-1} \widehat{\mathbf{A}}^{-1,1}-\widehat{\mathbf{A}}^{-1,1} \widehat{\mathbf{A}}^{1,-1}\right)=\widehat{\mathbf{D}},
$$

with

$$
\widehat{\mathbf{D}}=\frac{i\left|\boldsymbol{\kappa}_{\perp}\right|^{2}}{2 \omega}\left(\begin{array}{cccccc}
-c_{S} & 0 & 0 & 0 & 0 & 0 \\
0 & c_{S} & 0 & 0 & 0 & 0 \\
0 & 0 & -c_{S} & 0 & 0 & 0 \\
0 & 0 & 0 & c_{S} & 0 & 0 \\
0 & 0 & 0 & 0 & -c_{P} & 0 \\
0 & 0 & 0 & 0 & 0 & c_{P}
\end{array}\right)
$$

It is absolutely remarkable that the zero-th order term $\widehat{\mathbf{A}}^{0,0}$ and the Lie brackets coming from the averaging theorem combine to give a diagonal matrix, while each contribution is rather complicated. This gives the following equations for the reflection and transmission matrices:

$$
\begin{aligned}
& \frac{d \widehat{\mathbf{R}}}{d x_{3}}=\left(\mathbf{I}-\widehat{\mathbf{R}} \mathbf{H}^{0}\right) \widehat{\mathbf{D}} \widehat{\mathbf{R}}, \quad \widehat{\mathbf{R}}\left(x_{3}=-L\right)=\mathbf{I}, \\
& \frac{d \widehat{\mathbf{T}}}{d x_{3}}=-\widehat{\mathbf{T}} \mathbf{H}^{0} \widehat{\mathbf{D}} \widehat{\mathbf{R}}, \quad \widehat{\mathbf{T}}\left(x_{3}=-L\right)=\mathbf{I} .
\end{aligned}
$$

The solution of (80) is a diagonal matrix whose even diagonal terms are equal to one:

$$
\widehat{\mathbf{R}}\left(x_{3}\right)=\left(\begin{array}{cccccc}
\widehat{R}_{S}\left(x_{3}\right) & 0 & 0 & 0 & 0 & 0 \\
0 & 1 & 0 & 0 & 0 & 0 \\
0 & 0 & \widehat{R}_{S}\left(x_{3}\right) & 0 & 0 & 0 \\
0 & 0 & 0 & 1 & 0 & 0 \\
0 & 0 & 0 & 0 & \widehat{R}_{P}\left(x_{3}\right) & 0 \\
0 & 0 & 0 & 0 & 0 & 1
\end{array}\right)
$$

where 


$$
\begin{array}{cc}
\frac{d \widehat{R}_{S}}{d x_{3}}\left(x_{3}\right)=-\frac{i c_{S}\left|\boldsymbol{\kappa}_{\perp}\right|^{2}}{2 \omega} \widehat{R}_{S}\left(x_{3}\right), & \widehat{R}_{S}\left(x_{3}=-L\right)=1, \\
\frac{d \widehat{R}_{P}}{d x_{3}}\left(x_{3}\right)=-\frac{i c_{P}\left|\boldsymbol{\kappa}_{\perp}\right|^{2}}{2 \omega} \widehat{R}_{P}\left(x_{3}\right), & \widehat{R}_{P}\left(x_{3}=-L\right)=1 .
\end{array}
$$

Therefore, for $j=1,2,3$, we have

$$
\lim _{\varepsilon \rightarrow 0} \widehat{a}_{j}^{\varepsilon}\left(x_{3}=0\right)=\left[\widehat{\mathbf{R}}\left(x_{3}=0\right) \widehat{\boldsymbol{V}}\right]_{2 j-1}=0 .
$$

This shows that the paraxial (or forward-scattering) approximation is valid in the sense that the reflected modes $\widehat{a}_{j}^{\varepsilon}\left(x_{3}=0\right)$ are vanishing in the limit $\varepsilon \rightarrow 0$. The solution of $(81)$ is also a diagonal matrix:

$$
\widehat{\mathbf{T}}\left(x_{3}\right)=\left(\begin{array}{cccccc}
1 & 0 & 0 & 0 & 0 & 0 \\
0 & \widehat{T}_{S}\left(x_{3}\right) & 0 & 0 & 0 & 0 \\
0 & 0 & 1 & 0 & 0 & 0 \\
0 & 0 & 0 & \widehat{T}_{S}\left(x_{3}\right) & 0 & 0 \\
0 & 0 & 0 & 0 & 1 & 0 \\
0 & 0 & 0 & 0 & 0 & \widehat{T}_{P}\left(x_{3}\right)
\end{array}\right),
$$

where

$$
\begin{array}{ll}
\frac{d \widehat{T}_{S}}{d x_{3}}\left(x_{3}\right)=-\frac{i c_{S}\left|\boldsymbol{\kappa}_{\perp}\right|^{2}}{2 \omega} \widehat{T}_{S}\left(x_{3}\right), & \widehat{T}_{S}\left(x_{3}=-L\right)=1, \\
\frac{d \widehat{T}_{P}}{d x_{3}}\left(x_{3}\right)=-\frac{i c_{P}\left|\boldsymbol{\kappa}_{\perp}\right|^{2}}{2 \omega} \widehat{T}_{P}\left(x_{3}\right), & \widehat{T}_{P}\left(x_{3}=-L\right)=1 .
\end{array}
$$

By taking an inverse Fourier transform in $\boldsymbol{\kappa}_{\perp}$, we obtain the result of Proposition 1. Finally, the solutions of the Schrödinger equations in a homogeneous medium can be computed explicitly and we obtain

$$
\begin{aligned}
& \chi_{P 33}\left(s, \boldsymbol{x}_{\perp}\right)=\frac{\zeta_{P}^{1 / 2}}{(2 \pi)^{3}} \iint \check{b}_{\mathrm{inc}, 3}\left(\omega, \boldsymbol{\kappa}_{\perp}\right) e^{-\frac{i c_{P}}{2 \omega}\left|\boldsymbol{\kappa}_{\perp}\right|^{2} L+i \boldsymbol{\kappa}_{\perp} \cdot \boldsymbol{x}_{\perp}} d \boldsymbol{\kappa}_{\perp} e^{-i \omega s} d \omega \\
& \chi_{S j 3}\left(s, \boldsymbol{x}_{\perp}\right)=\frac{\zeta_{S}^{1 / 2}}{(2 \pi)^{3}} \iint \check{b}_{\mathrm{inc}, j}\left(\omega, \boldsymbol{\kappa}_{\perp}\right) e^{-\frac{i c_{S}}{2 \omega}\left|\boldsymbol{\kappa}_{\perp}\right|^{2} L+i \boldsymbol{\kappa}_{\perp} \cdot \boldsymbol{x}_{\perp}} d \boldsymbol{\kappa}_{\perp} e^{-i \omega s} d \omega, \quad j=1,2 .
\end{aligned}
$$

\section{The derivation of the paraxial wave equations in the random case}

In this section we prove Proposition 2. We transform the two-point boundary value problem (42)-(44) into an initial value problem. This is very important in the random case so that we can deal exclusively with quantities that are adapted to the filtration of the driving processes $m_{\mu}, m_{\rho}$, and $m_{\lambda}$ (which is necessary for the application of a diffusion approximation theorem). This transformation is done by an invariant imbedding step in which we introduce transmission and reflection operators. The algebra is more complicated than in the homogeneous case since the random medium fluctuations involve coupling not only between the six modes (as in the homogeneous case) but also between different $\boldsymbol{\kappa}_{\perp}$-modes. That is why we need to introduce matrix operators. The vector $\widehat{\boldsymbol{X}}^{\varepsilon}$ defined as in (41) is solution of the two-point boundary value problem:

$$
\frac{d \widehat{\boldsymbol{X}}^{\varepsilon}}{d x_{3}}=\widehat{\mathcal{A}}^{\varepsilon}\left(x_{3}\right) \widehat{\boldsymbol{X}}^{\varepsilon}, \quad \mathbf{H}^{-L} \widehat{\boldsymbol{X}}^{\varepsilon}(-L)+\mathbf{H}^{0} \widehat{\boldsymbol{X}}^{\varepsilon}(0)=\widehat{\boldsymbol{V}} .
$$

Here $\widehat{\mathcal{A}}^{\varepsilon}\left(x_{3}\right)$ is the matrix operator acting on six-dimensional vector fields $\widehat{\boldsymbol{Y}}\left(\boldsymbol{\kappa}_{\perp}\right)$ as

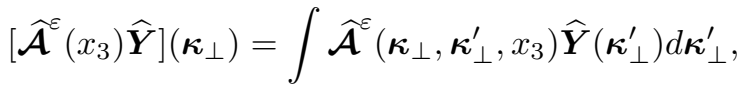

with the kernel 


$$
\begin{aligned}
\widehat{\mathcal{A}}^{\varepsilon}\left(\boldsymbol{\kappa}_{\perp}, \boldsymbol{\kappa}_{\perp}^{\prime}, x_{3}\right)= & \delta\left(\boldsymbol{\kappa}_{\perp}-\boldsymbol{\kappa}_{\perp}^{\prime}\right)\left\{\widehat{\mathbf{A}}^{0,0}+\frac{1}{\varepsilon^{2}}\left[e^{i \frac{\omega x_{3}}{\varepsilon^{4}}\left(\frac{1}{c_{P}}+\frac{1}{c_{S}}\right)} \widehat{\mathbf{A}}^{1,1}+e^{i \frac{\omega x_{3}}{\varepsilon^{4}}\left(\frac{1}{c_{P}}-\frac{1}{c_{S}}\right)} \widehat{\mathbf{A}}^{1,-1}\right.\right. \\
& \left.\left.+e^{i \frac{i x_{3}}{\varepsilon^{4}}\left(-\frac{1}{c_{P}}+\frac{1}{c_{S}}\right)} \widehat{\mathbf{A}}^{-1,1}+e^{i \frac{\omega x_{3}}{\varepsilon^{4}}\left(-\frac{1}{c_{P}}-\frac{1}{c_{S}}\right)} \widehat{\mathbf{A}}^{-1,-1}\right]\right\} \\
& +\frac{1}{\varepsilon} \frac{1}{(2 \pi)^{2}} \widehat{\tilde{m}}_{P}\left(\boldsymbol{\kappa}_{\perp}-\boldsymbol{\kappa}_{\perp}^{\prime}, \frac{x_{3}}{\varepsilon^{2}}\right)\left[e^{i \frac{2 \omega x_{3}}{\varepsilon^{4} c_{P}}} \widehat{\mathbf{B}}^{2,0}+e^{-i \frac{2 \omega x_{3}}{\varepsilon^{4} c_{P}}} \widehat{\mathbf{B}}^{-2,0}\right] \\
& +\frac{1}{\varepsilon} \frac{1}{(2 \pi)^{2}} \widehat{\tilde{m}}_{S}\left(\boldsymbol{\kappa}_{\perp}-\boldsymbol{\kappa}_{\perp}^{\prime}, \frac{x_{3}}{\varepsilon^{2}}\right)\left[e^{i \frac{2 \omega x_{3}}{\varepsilon^{4} c_{S}}} \widehat{\mathbf{B}}^{0,2}+e^{-i \frac{2 \omega x_{3}}{\varepsilon^{4} c_{S}}} \widehat{\mathbf{B}}^{0,-2}\right] \\
& +\frac{1}{\varepsilon} \frac{1}{(2 \pi)^{2}} \widehat{m}_{P}\left(\boldsymbol{\kappa}_{\perp}-\boldsymbol{\kappa}_{\perp}^{\prime}, \frac{x_{3}}{\varepsilon^{2}}\right) \widehat{\mathbf{B}}_{P}^{0,0}+\frac{1}{\varepsilon} \frac{1}{(2 \pi)^{2}} \widehat{m}_{S}\left(\boldsymbol{\kappa}_{\perp}-\boldsymbol{\kappa}_{\perp}^{\prime}, \frac{x_{3}}{\varepsilon^{2}}\right) \widehat{\mathbf{B}}_{S}^{0,0} .
\end{aligned}
$$

By applying Proposition B.1, we get that the reflected and transmitted modes are given by

$$
\begin{array}{ll}
\widehat{a}_{j}^{\varepsilon}\left(x_{3}=0\right)=\left[\widehat{\mathcal{R}}^{\varepsilon}\left(x_{3}=0\right) \widehat{\boldsymbol{V}}\right]_{2 j-1}, & j=1,2,3, \\
\widehat{b}_{j}^{\varepsilon}\left(x_{3}=-L\right)=\left[\widehat{\mathcal{T}}^{\varepsilon}\left(x_{3}=0\right) \widehat{\boldsymbol{V}}\right]_{2 j}, & j=1,2,3,
\end{array}
$$

where the reflection and transmission matrix-operator kernels are solution of the initial value problem

$$
\begin{aligned}
& \frac{d \widehat{\mathcal{R}}^{\varepsilon}}{d x_{3}}=\left(\widehat{\mathcal{I}}-\widehat{\mathcal{\mathcal { R }}}^{\varepsilon} \widehat{\mathcal{H}}^{0}\right) \widehat{\mathcal{\mathcal { A }}}^{\varepsilon}\left(x_{3}\right) \widehat{\mathcal{\mathcal { R }}}^{\varepsilon}, \quad \widehat{\mathcal{\mathcal { R }}}^{\varepsilon}\left(x_{3}=-L\right)=\widehat{\mathcal{I}}, \\
& \frac{d \widehat{\mathcal{\mathcal { T }}}^{\varepsilon}}{d x_{3}}=-\widehat{\mathcal{\mathcal { T }}}^{\varepsilon} \widehat{\mathcal{H}}^{0} \widehat{\mathcal{A}}^{\varepsilon}\left(x_{3}\right) \widehat{\mathcal{\mathcal { R }}}^{\varepsilon}, \quad \widehat{\mathcal{I}}^{\varepsilon}\left(x_{3}=-L\right)=\widehat{\mathcal{I}},
\end{aligned}
$$

where

$$
\widehat{\mathcal{I}}\left(\boldsymbol{\kappa}_{\perp}, \boldsymbol{\kappa}_{\perp}^{\prime}\right)=\delta\left(\boldsymbol{\kappa}_{\perp}-\boldsymbol{\kappa}_{\perp}^{\prime}\right) \mathbf{I}, \quad \widehat{\mathcal{H}}^{0}\left(\boldsymbol{\kappa}_{\perp}, \boldsymbol{\kappa}_{\perp}^{\prime}\right)=\delta\left(\boldsymbol{\kappa}_{\perp}-\boldsymbol{\kappa}_{\perp}^{\prime}\right) \mathbf{H}^{0} .
$$

Explicitly, the transmitted wave components are, for $j=1,2$ :

$$
\begin{gathered}
\chi_{j 3}\left(t, \varepsilon^{2} \boldsymbol{x}_{\perp}, x_{3}=-L\right)=\frac{\zeta_{S}^{1 / 2}}{(2 \pi)^{3}} \iint \sum_{k=1}^{3} \widehat{\mathcal{T}}_{2 j, 2 k}^{\varepsilon}\left(\omega, \boldsymbol{\kappa}_{\perp}, \boldsymbol{\kappa}_{\perp}^{\prime}, x_{3}=0\right) \widehat{b}_{\mathrm{inc}, k}\left(\omega, \boldsymbol{\kappa}_{\perp}^{\prime}\right) e^{i \boldsymbol{\kappa}_{\perp} \cdot \boldsymbol{x}_{\perp}} d \boldsymbol{\kappa}_{\perp}^{\prime} d \boldsymbol{\kappa}_{\perp} e^{i \frac{\omega}{\varepsilon 4}\left(\frac{L}{c_{S}}-t\right)} d \omega, \\
\chi_{33}\left(t, \varepsilon^{2} \boldsymbol{x}_{\perp}, x_{3}=-L\right)=\frac{\zeta_{P}^{1 / 2}}{(2 \pi)^{3}} \iint \sum_{k=1}^{3} \widehat{\mathcal{T}}_{6,2 k}^{\varepsilon}\left(\omega, \boldsymbol{\kappa}_{\perp}, \boldsymbol{\kappa}_{\perp}^{\prime}, x_{3}=0\right) \widehat{b}_{\mathrm{inc}, k}\left(\omega, \boldsymbol{\kappa}_{\perp}^{\prime}\right) e^{i \boldsymbol{\kappa}_{\perp} \cdot \boldsymbol{x}_{\perp}} d \boldsymbol{\kappa}_{\perp}^{\prime} d \boldsymbol{\kappa}_{\perp} e^{i \frac{\omega}{\varepsilon 4}\left(\frac{L}{c_{P}}-t\right)} d \omega,
\end{gathered}
$$

$\xi_{j}=\zeta_{S}^{-1} \chi_{j 3}$ and $\xi_{3}=\zeta_{P}^{-1} \chi_{33}$ while the other components are obtained by (19-22). We first note that the rapid phase in $\omega$ will give a localization in time of the transmitted waves (provided we show that $\hat{\mathcal{T}}^{\varepsilon}$ has a limit). Therefore we focus our attention on

$$
\begin{aligned}
& \chi_{j 3}^{\varepsilon}\left(s, \boldsymbol{x}_{\perp}\right)=\chi_{j 3}\left(t=\frac{L}{c_{S}}+\varepsilon^{4} s, \varepsilon^{2} \boldsymbol{x}_{\perp}, x_{3}=-L\right), \\
& \chi_{33}^{\varepsilon}\left(s, \boldsymbol{x}_{\perp}\right)=\chi_{33}\left(t=\frac{L}{c_{P}}+\varepsilon^{4} s, \varepsilon^{2} \boldsymbol{x}_{\perp}, x_{3}=-L\right) .
\end{aligned}
$$

The proof of the convergence follows closely the strategy adopted in [14], where the paraxial wave equation is obtained from the acoustic wave equations in the same distinguished limit. The proof is similar because the matrix operator $\widehat{\mathcal{A}}^{\varepsilon}$ has a diagonal block structure (with $2 \times 2$ blocks along the diagonal), which means that it has the same structure as the $2 \times 2$ operator encountered in the acoustic case. The main step of the proof consists in showing the convergence of the general moments of the transmitted wave components to the ones given by the limit system of stochastic partial differential equation (64-65). For $N \in \mathbb{N}, j_{r} \in\{1,2,3\}$, $m_{r} \in \mathbb{N}, s_{r} \in \mathbb{R}$ and $\boldsymbol{x}_{\perp r} \in \mathbb{R}^{2}, r=1, \ldots, N$, the general moment of transmitted wave components

$$
I^{\varepsilon}=\mathbb{E}\left[\prod_{r=1}^{N} \chi_{j_{r} 3}^{\varepsilon}\left(s_{r}, \boldsymbol{x}_{\perp r}\right)^{m_{r}}\right]
$$

can be expressed as a sum of $3^{M}$ multiple integrals, for $M=\sum_{r=1}^{N} m_{r}$ : 


$$
\begin{aligned}
I^{\varepsilon}= & \sum_{\boldsymbol{k}=\left(k_{r, h}\right)_{h=1, \ldots, m_{r}, r=1, \ldots, N} \in\{1,2,3\}^{M}} I_{\boldsymbol{k}}^{\varepsilon}, \\
I_{\boldsymbol{k}}^{\varepsilon}= & \frac{\zeta_{S}^{\frac{M_{S}}{2}} \zeta_{P}^{\frac{M_{P}}{2}}}{(2 \pi)^{3 M}} \int \cdots \int \prod_{r=1}^{N} \prod_{h=1}^{m_{r}} d \boldsymbol{\kappa}_{\perp_{r, h}}^{\prime} d \boldsymbol{\kappa}_{\perp r, h} d \omega_{r, h} \\
& \times \prod_{r, h}\left(\widehat{b}_{\mathrm{inc}, k_{r, h}}\left(\omega_{r, h}, \boldsymbol{\kappa}_{\perp r, h}^{\prime}\right) e^{i\left(\boldsymbol{\kappa}_{\perp r, h} \cdot \boldsymbol{x}_{\perp r}-\omega_{r, h} s_{r}\right)}\right) \mathbb{E}\left[\prod_{r, h} \widehat{\mathcal{T}}_{2 j_{r}, 2 k_{r, h}}^{\varepsilon}\left(\omega_{r, h}, \boldsymbol{\kappa}_{\perp r, h}, \boldsymbol{\kappa}_{\perp r, h}^{\prime}, 0\right)\right],
\end{aligned}
$$

for $M_{P}=\operatorname{card}\left\{k_{r, h}=3, h=1, \ldots, m_{r}, r=1, \ldots, N\right\}$ and $M_{S}=M-M_{P}$. Therefore, the convergence of the general moment of the transmitted wave components in the white-noise limit will follow from the convergence of the specific moments $\mathbb{E}\left[J^{\varepsilon}(0)\right]$ of the transmission matrix-operator kernels, where

$$
J^{\varepsilon}\left(x_{3}\right)=\prod_{r=1}^{M} \widehat{\mathcal{T}}_{j_{r}, k_{r}}^{\varepsilon}\left(\omega_{r}, \boldsymbol{\kappa}_{\perp_{r}}, \boldsymbol{\kappa}_{\perp_{r}}^{\prime}, x_{3}\right) .
$$

We call these moments "specific" because we restrict our attention to the case in which the frequencies $\omega_{r}$, $r=1, \ldots, N$, are all distinct in (87). It is important to note that the transmission operator kernels $\widehat{\mathcal{T}}^{\varepsilon}$ themselves do not converge to $\widehat{\mathcal{T}}$, but only certain moments (expectations of products of components with distinct frequencies), which are those needed to ensure the convergence of the transmitted fields.

We use diffusion approximation theorems in the same way as in [14] combined with the application of the averaging theorem in Appendix $\mathrm{C}$ as in the homogeneous case to obtain the limit of the expectation of $J^{\varepsilon}(0)$ as $\varepsilon \rightarrow 0$. We find that this limit can be expressed as the expectation of the corresponding product of components of an "effective" transmission kernel in the following way:

$$
\lim _{\varepsilon \rightarrow 0} \mathbb{E}\left[J^{\varepsilon}(0)\right]=\mathbb{E}\left[\prod_{r=1}^{M} \widehat{\mathcal{T}}_{j_{r}, k_{r}}\left(\omega_{r}, \boldsymbol{\kappa}_{\perp r}, \boldsymbol{\kappa}_{\perp r}^{\prime}, 0\right)\right] .
$$

The expectation on the right-hand side is taken with respect to the following Itô-Schrödinger model for the transmission operator:

$$
\widehat{\mathcal{T}}\left(\omega, \boldsymbol{\kappa}_{\perp}, \boldsymbol{\kappa}_{\perp}^{\prime}, x_{3}\right)=\left(\begin{array}{ccccccc}
\delta\left(\boldsymbol{\kappa}_{\perp}-\boldsymbol{\kappa}_{\perp}^{\prime}\right) & 0 & 0 & 0 & 0 & 0 \\
0 & \widehat{\mathcal{T}}_{S} & 0 & 0 & 0 & 0 \\
0 & 0 & \delta\left(\boldsymbol{\kappa}_{\perp}-\boldsymbol{\kappa}_{\perp}^{\prime}\right) & 0 & 0 & 0 \\
0 & 0 & 0 & \widehat{\mathcal{T}}_{S} & 0 & 0 \\
0 & 0 & 0 & 0 & \delta\left(\boldsymbol{\kappa}_{\perp}-\boldsymbol{\kappa}_{\perp}^{\prime}\right) & 0 \\
0 & 0 & 0 & 0 & 0 & \widehat{\mathcal{T}}_{P}
\end{array}\right),
$$

where the kernels $\widehat{\mathcal{T}}_{S}\left(\omega, \boldsymbol{\kappa}_{\perp}, \boldsymbol{\kappa}_{\perp}^{\prime}, x_{3}\right)$ and $\widehat{\mathcal{T}}_{P}\left(\omega, \boldsymbol{\kappa}_{\perp}, \boldsymbol{\kappa}_{\perp}^{\prime}, x_{3}\right)$ are solution of

$$
\begin{aligned}
d \widehat{\mathcal{T}}_{S}\left(\omega, \boldsymbol{\kappa}_{\perp}, \boldsymbol{\kappa}_{\perp}^{\prime}, x_{3}\right)= & -\frac{i c_{S}\left|\boldsymbol{\kappa}_{\perp}^{\prime}\right|^{2}}{2 \omega} \check{\mathcal{T}}_{S}\left(\omega, \boldsymbol{\kappa}_{\perp}, \boldsymbol{\kappa}_{\perp}^{\prime}, x_{3}\right) d x_{3}-\frac{\omega^{2} C_{0, S S}(\mathbf{0})}{8 c_{S}^{2}} \widehat{\mathcal{T}}_{S}\left(\omega, \boldsymbol{\kappa}_{\perp}, \boldsymbol{\kappa}_{\perp}^{\prime}, x_{3}\right) d x_{3} \\
& +\frac{i \omega}{2(2 \pi)^{2} c_{S}} \int \widehat{\mathcal{T}}_{S}\left(\omega, \boldsymbol{\kappa}_{\perp}, \boldsymbol{\kappa}_{\perp}^{\prime \prime}, x_{3}\right) d \widehat{B}_{S}\left(\boldsymbol{\kappa}_{\perp}^{\prime \prime}-\boldsymbol{\kappa}_{\perp}^{\prime}, x_{3}\right) d \boldsymbol{\kappa}_{\perp}^{\prime \prime}, \\
d \widehat{\mathcal{T}}_{P}\left(\omega, \boldsymbol{\kappa}_{\perp}, \boldsymbol{\kappa}_{\perp}^{\prime}, x_{3}\right)= & -\frac{i c_{P}\left|\boldsymbol{\kappa}_{\perp}^{\prime}\right|^{2}}{2 \omega} \widehat{\mathcal{T}}_{P}\left(\omega, \boldsymbol{\kappa}_{\perp}, \boldsymbol{\kappa}_{\perp}^{\prime}, x_{3}\right) d x_{3}-\frac{\omega^{2} C_{0, P P}(\mathbf{0})}{8 c_{P}^{2}} \widehat{\mathcal{T}}_{P}\left(\omega, \boldsymbol{\kappa}_{\perp}, \boldsymbol{\kappa}_{\perp}^{\prime}, x_{3}\right) d x_{3} \\
& +\frac{i \omega}{2(2 \pi)^{2} c_{P}} \int \widehat{\mathcal{T}}_{P}\left(\omega, \boldsymbol{\kappa}_{\perp}, \boldsymbol{\kappa}_{\perp}^{\prime \prime}, x_{3}\right) d \widehat{B}_{P}\left(\boldsymbol{\kappa}_{\perp}^{\prime \prime}-\boldsymbol{\kappa}_{\perp}^{\prime}, x_{3}\right) d \boldsymbol{\kappa}_{\perp}^{\prime \prime},
\end{aligned}
$$

starting from $\widehat{\mathcal{T}}_{S}\left(\omega, \boldsymbol{\kappa}_{\perp}, \boldsymbol{\kappa}_{\perp}^{\prime}, x_{3}=-L\right)=\delta\left(\boldsymbol{\kappa}_{\perp}-\boldsymbol{\kappa}_{\perp}^{\prime}\right)$ and $\widehat{\mathcal{T}}_{P}\left(\omega, \boldsymbol{\kappa}_{\perp}, \boldsymbol{\kappa}_{\perp}^{\prime}, x_{3}=-L\right)=\delta\left(\boldsymbol{\kappa}_{\perp}-\boldsymbol{\kappa}_{\perp}^{\prime}\right)$. Here we use the standard Itô stochastic integral and the Brownian field $\widehat{\boldsymbol{B}}=\left(\widehat{B}_{S}, \widehat{B}_{P}\right)^{T}$ is the partial Fourier transform of the field $\boldsymbol{B}=\left(B_{S}, B_{P}\right)^{T}$. It has the following operator-valued spatial covariance:

$$
\mathbb{E}\left[\widehat{\boldsymbol{B}}\left(\boldsymbol{\kappa}_{\perp}, x_{3}\right) \widehat{\boldsymbol{B}}^{T}\left(\boldsymbol{\kappa}_{\perp}^{\prime}, x_{3}^{\prime}\right)\right]=\min \left\{x_{3}, x_{3}^{\prime}\right\}(2 \pi)^{2} \widehat{\mathbf{C}}_{0}\left(\boldsymbol{\kappa}_{\perp}\right) \delta\left(\boldsymbol{\kappa}_{\perp}+\boldsymbol{\kappa}_{\perp}^{\prime}\right),
$$


where

$$
\widehat{\mathbf{C}}_{0}\left(\boldsymbol{\kappa}_{\perp}\right)=\int_{\mathbb{R}^{2}} \mathbf{C}_{0}\left(\boldsymbol{x}_{\perp}\right) e^{-i \boldsymbol{\kappa}_{\perp} \cdot \boldsymbol{x}_{\perp}} d \boldsymbol{x}_{\perp} .
$$

By considering the transmission kernels in the original spatial variables:

$$
\begin{aligned}
& \check{\mathcal{T}}_{S}\left(\omega, \boldsymbol{x}_{\perp}, \boldsymbol{x}_{\perp}^{\prime}, x_{3}\right)=\frac{1}{(2 \pi)^{2}} \iint e^{i\left(\boldsymbol{\kappa}_{\perp} \cdot \boldsymbol{x}_{\perp}-\boldsymbol{\kappa}_{\perp}^{\prime} \cdot \boldsymbol{x}_{\perp}^{\prime}\right)} \widehat{\mathcal{T}}_{S}\left(\omega, \boldsymbol{\kappa}_{\perp}, \boldsymbol{\kappa}_{\perp}^{\prime}, x_{3}\right) d \boldsymbol{\kappa}_{\perp} d \boldsymbol{\kappa}_{\perp}^{\prime}, \\
& \check{\mathcal{T}}_{P}\left(\omega, \boldsymbol{x}_{\perp}, \boldsymbol{x}_{\perp}^{\prime}, x_{3}\right)=\frac{1}{(2 \pi)^{2}} \iint e^{i\left(\boldsymbol{\kappa}_{\perp} \cdot \boldsymbol{x}_{\perp}-\boldsymbol{\kappa}_{\perp}^{\prime} \cdot \boldsymbol{x}_{\perp}^{\prime}\right)} \widehat{\mathcal{T}}_{P}\left(\omega, \boldsymbol{\kappa}_{\perp}, \boldsymbol{\kappa}_{\perp}^{\prime}, x_{3}\right) d \boldsymbol{\kappa}_{\perp} d \boldsymbol{\kappa}_{\perp}^{\prime},
\end{aligned}
$$

we obtain that they satisfy the system (64-65) where we have used the Stratonovich integral instead of Itô integral.

\section{Conclusion}

In this paper we have used invariant imbedding and limit theorems to obtain the paraxial equations for elastic waves in random media in the parabolic white-noise regime. We have shown that the paraxial systems for the pressure waves and shear waves have the form of a system of Schrödinger equations that are driven by a pair of correlated Brownian fields. We have identified the structure of the correlation between the Brownian fields and between the wave fields and we have clarified the form of the diffraction operators. These equations give a new and efficient way of modeling elastic wave propagation in a consistent manner via averaging and diffusion approximation results. Owing to the central role elastic wave propagation have in for instance geophysics we expect these result to be relevant in a number of applications, in particular for analyzing schemes for imaging theoretically as well as for numerical wave propagation.

\section{Appendix A. Expressions of the matrices}

The matrices $\check{\mathbf{A}}$ are obtained from $\widehat{\mathbf{A}}$ by substitution $i \kappa_{1} \rightarrow \frac{\partial}{\partial x_{1}}$ and $i \kappa_{2} \rightarrow \frac{\partial}{\partial x_{2}}$.

$$
\begin{gathered}
\widehat{\mathbf{A}}^{1,1}=\left(\begin{array}{cccccc}
0 & 0 & 0 & 0 & 0 & 0 \\
0 & 0 & 0 & 0 & i \kappa_{1} \alpha_{a} & 0 \\
0 & 0 & 0 & 0 & 0 & 0 \\
0 & 0 & 0 & 0 & i \kappa_{2} \alpha_{a} & 0 \\
0 & 0 & 0 & 0 & 0 & 0 \\
i \kappa_{1} \alpha_{c} & 0 & i \kappa_{2} \alpha_{c} & 0 & 0 & 0
\end{array}\right), \quad \widehat{\mathbf{A}}^{-1,-1}=\left(\begin{array}{cccccc}
0 & 0 & 0 & 0 & 0 & i \kappa_{1} \alpha_{a} \\
0 & 0 & 0 & 0 & 0 & 0 \\
0 & 0 & 0 & 0 & 0 & i \kappa_{2} \alpha_{a} \\
0 & 0 & 0 & 0 & 0 & 0 \\
0 & i \kappa_{1} \alpha_{c} & 0 & i \kappa_{2} \alpha_{c} & 0 & 0 \\
0 & 0 & 0 & 0 & 0 & 0
\end{array}\right), \\
\widehat{\mathbf{A}}^{1,-1}=-\left(\begin{array}{lllllll}
0 & 0 & 0 & 0 & i \kappa_{1} \alpha_{b} & 0 \\
0 & 0 & 0 & 0 & 0 & 0 \\
0 & 0 & 0 & 0 & i \kappa_{2} \alpha_{b} & 0 \\
0 & 0 & 0 & 0 & 0 & 0 \\
0 & 0 & 0 & 0 & 0 & 0 \\
0 & i \kappa_{1} \alpha_{c} & 0 & i \kappa_{2} \alpha_{c} & 0 & 0
\end{array}\right), \quad \widehat{\mathbf{A}}^{-1,1}=-\left(\begin{array}{ccccccc}
0 & 0 & 0 & 0 & 0 & 0 \\
0 & 0 & 0 & 0 & 0 & i \kappa_{1} \alpha_{b} \\
0 & 0 & 0 & 0 & 0 & 0 \\
0 & 0 & 0 & 0 & 0 & i \kappa_{2} \alpha_{b} \\
i \kappa_{1} \alpha_{c} & 0 & i \kappa_{2} \alpha_{c} & 0 & 0 & 0 \\
0 & 0 & 0 & 0 & 0 & 0
\end{array}\right),
\end{gathered}
$$

with $\alpha_{a}=\frac{\zeta_{P}^{1 / 2}}{\zeta_{S}^{1 / 2}}\left(\frac{\lambda_{0}}{4 \mu_{0}}-\frac{\zeta_{S}}{2 \zeta_{P}}\right), \alpha_{b}=\frac{\zeta_{P}^{1 / 2}}{\zeta_{S}^{1 / 2}}\left(\frac{\lambda_{0}}{4 \mu_{0}}+\frac{\zeta_{S}}{2 \zeta_{P}}\right)$, and $\alpha_{c}=\frac{\zeta_{S}^{1 / 2}}{\zeta_{P}^{1 / 2}} \frac{\mu_{0}+\lambda_{0}}{2 \mu_{0}+\lambda_{0}}$, 


$$
\begin{aligned}
& \widehat{\mathbf{A}}^{0,0}=-\frac{i c_{S}\left|\boldsymbol{\kappa}_{\perp}\right|^{2}}{2 \omega}\left(\begin{array}{cccccc}
1 & 0 & 0 & 0 & 0 & 0 \\
0 & -1 & 0 & 0 & 0 & 0 \\
0 & 0 & 1 & 0 & 0 & 0 \\
0 & 0 & 0 & -1 & 0 & 0 \\
0 & 0 & 0 & 0 & 0 & 0 \\
0 & 0 & 0 & 0 & 0 & 0
\end{array}\right)-\frac{i c_{S}}{2 \omega} \frac{\mu_{0}+\lambda_{0}}{\mu_{0}}\left(\begin{array}{cccccc}
\kappa_{1}^{2} & 0 & \kappa_{1} \kappa_{2} & 0 & 0 & 0 \\
0 & -\kappa_{1}^{2} & 0 & -\kappa_{1} \kappa_{2} & 0 & 0 \\
\kappa_{1} \kappa_{2} & 0 & \kappa_{2}^{2} & 0 & 0 & 0 \\
0 & -\kappa_{1} \kappa_{2} & 0 & -\kappa_{2}^{2} & 0 & 0 \\
0 & 0 & 0 & 0 & 0 & 0 \\
0 & 0 & 0 & 0 & 0 & 0
\end{array}\right) \\
& -\frac{i c_{P}\left|\boldsymbol{\kappa}_{\perp}\right|^{2}}{2 \omega} \frac{\lambda_{0}}{2 \mu_{0}+\lambda_{0}}\left(\begin{array}{cccccc}
0 & 0 & 0 & 0 & 0 & 0 \\
0 & 0 & 0 & 0 & 0 & 0 \\
0 & 0 & 0 & 0 & 0 & 0 \\
0 & 0 & 0 & 0 & 0 & 0 \\
0 & 0 & 0 & 0 & -1 & 0 \\
0 & 0 & 0 & 0 & 0 & 1
\end{array}\right) \\
& \widehat{\mathbf{A}}^{0,2}=\frac{i c_{S}\left|\boldsymbol{\kappa}_{\perp}\right|^{2}}{2 \omega}\left(\begin{array}{llllll}
0 & 0 & 0 & 0 & 0 & 0 \\
1 & 0 & 0 & 0 & 0 & 0 \\
0 & 0 & 0 & 0 & 0 & 0 \\
0 & 0 & 1 & 0 & 0 & 0 \\
0 & 0 & 0 & 0 & 0 & 0 \\
0 & 0 & 0 & 0 & 0 & 0
\end{array}\right)+\frac{i c_{S}}{2 \omega} \frac{\mu_{0}+\lambda_{0}}{\mu_{0}}\left(\begin{array}{cccccc}
0 & 0 & 0 & 0 & 0 & 0 \\
\kappa_{1}^{2} & 0 & \kappa_{1} \kappa_{2} & 0 & 0 & 0 \\
0 & 0 & 0 & 0 & 0 & 0 \\
\kappa_{1} \kappa_{2} & 0 & \kappa_{2}^{2} & 0 & 0 & 0 \\
0 & 0 & 0 & 0 & 0 & 0 \\
0 & 0 & 0 & 0 & 0 & 0
\end{array}\right), \\
& \widehat{\mathbf{A}}^{0,-2}=-\frac{i c_{S}\left|\boldsymbol{\kappa}_{\perp}\right|^{2}}{2 \omega}\left(\begin{array}{cccccc}
0 & 1 & 0 & 0 & 0 & 0 \\
0 & 0 & 0 & 0 & 0 & 0 \\
0 & 0 & 0 & 1 & 0 & 0 \\
0 & 0 & 0 & 0 & 0 & 0 \\
0 & 0 & 0 & 0 & 0 & 0 \\
0 & 0 & 0 & 0 & 0 & 0
\end{array}\right)-\frac{i c_{S}}{2 \omega} \frac{\mu_{0}+\lambda_{0}}{\mu_{0}}\left(\begin{array}{ccccccc}
0 & \kappa_{1}^{2} & 0 & \kappa_{1} & \kappa_{2} & 0 & 0 \\
0 & 0 & 0 & 0 & 0 & 0 \\
0 & \kappa_{1} \kappa_{2} & 0 & \kappa_{2}^{2} & 0 & 0 \\
0 & 0 & 0 & 0 & 0 & 0 \\
0 & 0 & 0 & 0 & 0 & 0 \\
0 & 0 & 0 & 0 & 0 & 0
\end{array}\right), \\
& \widehat{\mathbf{A}}^{2,0}=-\frac{i c_{P}\left|\boldsymbol{\kappa}_{\perp}\right|^{2}}{2 \omega} \frac{\lambda_{0}}{2 \mu_{0}+\lambda_{0}}\left(\begin{array}{llllll}
0 & 0 & 0 & 0 & 0 & 0 \\
0 & 0 & 0 & 0 & 0 & 0 \\
0 & 0 & 0 & 0 & 0 & 0 \\
0 & 0 & 0 & 0 & 0 & 0 \\
0 & 0 & 0 & 0 & 0 & 0 \\
0 & 0 & 0 & 0 & 1 & 0
\end{array}\right), \quad \widehat{\mathbf{A}}^{-2,0}=\frac{i c_{P}\left|\boldsymbol{\kappa}_{\perp}\right|^{2}}{2 \omega} \frac{\lambda_{0}}{2 \mu_{0}+\lambda_{0}}\left(\begin{array}{llllll}
0 & 0 & 0 & 0 & 0 & 0 \\
0 & 0 & 0 & 0 & 0 & 0 \\
0 & 0 & 0 & 0 & 0 & 0 \\
0 & 0 & 0 & 0 & 0 & 0 \\
0 & 0 & 0 & 0 & 0 & 1 \\
0 & 0 & 0 & 0 & 0 & 0
\end{array}\right), \\
& \widehat{\mathbf{B}}^{0,2}=\frac{i \omega}{2 c_{S}}\left(\begin{array}{cccccc}
0 & 0 & 0 & 0 & 0 & 0 \\
1 & 0 & 0 & 0 & 0 & 0 \\
0 & 0 & 0 & 0 & 0 & 0 \\
0 & 0 & 1 & 0 & 0 & 0 \\
0 & 0 & 0 & 0 & 0 & 0 \\
0 & 0 & 0 & 0 & 0 & 0
\end{array}\right), \quad \widehat{\mathbf{B}}^{0,-2}=-\frac{i \omega}{2 c_{S}}\left(\begin{array}{llllll}
0 & 1 & 0 & 0 & 0 & 0 \\
0 & 0 & 0 & 0 & 0 & 0 \\
0 & 0 & 0 & 1 & 0 & 0 \\
0 & 0 & 0 & 0 & 0 & 0 \\
0 & 0 & 0 & 0 & 0 & 0 \\
0 & 0 & 0 & 0 & 0 & 0
\end{array}\right), \\
& \widehat{\mathbf{B}}^{2,0}=\frac{i \omega}{2 c_{P}}\left(\begin{array}{llllll}
0 & 0 & 0 & 0 & 0 & 0 \\
0 & 0 & 0 & 0 & 0 & 0 \\
0 & 0 & 0 & 0 & 0 & 0 \\
0 & 0 & 0 & 0 & 0 & 0 \\
0 & 0 & 0 & 0 & 0 & 0 \\
0 & 0 & 0 & 0 & 1 & 0
\end{array}\right), \quad \widehat{\mathbf{B}}^{-2,0}=-\frac{i \omega}{2 c_{P}}\left(\begin{array}{llllll}
0 & 0 & 0 & 0 & 0 & 0 \\
0 & 0 & 0 & 0 & 0 & 0 \\
0 & 0 & 0 & 0 & 0 & 0 \\
0 & 0 & 0 & 0 & 0 & 0 \\
0 & 0 & 0 & 0 & 0 & 1 \\
0 & 0 & 0 & 0 & 0 & 0
\end{array}\right), \\
& \widehat{\mathbf{B}}_{S}^{0,0}=\frac{i \omega}{2 c_{S}}\left(\begin{array}{cccccc}
1 & 0 & 0 & 0 & 0 & 0 \\
0 & -1 & 0 & 0 & 0 & 0 \\
0 & 0 & 1 & 0 & 0 & 0 \\
0 & 0 & 0 & -1 & 0 & 0 \\
0 & 0 & 0 & 0 & 0 & 0 \\
0 & 0 & 0 & 0 & 0 & 0
\end{array}\right), \quad \widehat{\mathbf{B}}_{P}^{0,0}=\frac{i \omega}{2 c_{P}}\left(\begin{array}{cccccc}
0 & 0 & 0 & 0 & 0 & 0 \\
0 & 0 & 0 & 0 & 0 & 0 \\
0 & 0 & 0 & 0 & 0 & 0 \\
0 & 0 & 0 & 0 & 0 & 0 \\
0 & 0 & 0 & 0 & 1 & 0 \\
0 & 0 & 0 & 0 & 0 & -1
\end{array}\right) .
\end{aligned}
$$




\section{Appendix B. An invariant imbedding theorem}

Let us consider the two-point boundary value problem:

$$
\frac{d \boldsymbol{X}}{d z}(z)=\mathbf{A}(z) \boldsymbol{X}(z), \quad \boldsymbol{X}(z) \in \mathbb{R}^{m},
$$

with the boundary condition $\mathbf{H}^{-L} \boldsymbol{X}(-L)+\mathbf{H}^{0} \boldsymbol{X}(0)=\boldsymbol{V}^{0}$, where $\mathbf{A}(z), \mathbf{H}^{-L}$ and $\mathbf{H}^{0}$ are $m \times m$-matrices and $\boldsymbol{V}^{0}$ is an $m$-dimensional vector. Assume that $\mathbf{H}^{-L}+\mathbf{H}^{0}$ is invertible. In this linear framework the invariant imbedding approach leads to the following proposition [3].

Proposition B.1 Let us define the matrices $(\mathbf{R}(\zeta))_{-L \leq \zeta \leq 0}$ and $(\mathbf{Q}(z, \zeta))_{-L \leq z \leq \zeta \leq 0}$ as the solutions of the initial value problems:

$$
\frac{d \mathbf{R}}{d \zeta}(\zeta)=\mathbf{A}(\zeta) \mathbf{R}(\zeta)-\mathbf{R}(\zeta) \mathbf{H}^{0} \mathbf{A}(\zeta) \mathbf{R}(\zeta), \quad-L \leq \zeta \leq 0,
$$

starting from $\zeta=-L: \mathbf{R}(\zeta=-L)=\left(\mathbf{H}^{-L}+\mathbf{H}^{0}\right)^{-1}$, and

$$
\frac{\partial \mathbf{Q}}{\partial \zeta}(z, \zeta)=-\mathbf{Q}(z, \zeta) \mathbf{H}^{0} \mathbf{A}(\zeta) \mathbf{R}(\zeta), \quad-L \leq z \leq \zeta \leq 0,
$$

starting from $\zeta=z: \mathbf{Q}(z, \zeta=z)=\mathbf{R}(z)$. If the solution $\mathbf{R}(\zeta)$ of the nonlinear initial value problem (B.2) exists up to $\zeta=0$, then $\mathbf{P}(z)=\mathbf{Q}(z, 0)$ is the solution of:

$$
\frac{d \mathbf{P}}{d z}(z)=\mathbf{A}(z) \mathbf{P}(z), \quad 0 \leq z \leq L, \quad \text { with } \mathbf{H}^{-L} \mathbf{P}(-L)+\mathbf{H}^{0} \mathbf{P}(0)=\mathbf{I},
$$

and consequently $\boldsymbol{X}(z)=\mathbf{P}(z) \boldsymbol{V}^{0}$ is the solution of (B.1) with the boundary condition $\mathbf{H}^{-L} \boldsymbol{X}(-L)+$ $\mathbf{H}^{0} \boldsymbol{X}(0)=\boldsymbol{V}^{0}$.

\section{Appendix C. An averaging theorem}

The following proposition can be found in [21,23]. We give here an elementary proof for consistency.

Proposition C.1 Let us consider the solution $\mathbf{X}^{\varepsilon}=\left(X_{j k}^{\varepsilon}\right)_{j, k=1, \ldots, N}$ of the initial value problem

$$
\frac{d \mathbf{X}^{\varepsilon}}{d z}=\mathbf{F}^{(0)}\left(\mathbf{X}^{\varepsilon}\right)+\frac{1}{\varepsilon^{2}} \sum_{q=1}^{Q}\left(\mathbf{F}^{(q)}\left(\mathbf{X}^{\varepsilon}\right) e^{i \frac{\alpha^{(q) z}}{\varepsilon^{4}}}+\mathbf{G}^{(q)}\left(\mathbf{X}^{\varepsilon}\right) e^{-i \frac{\alpha^{(q)} z}{\varepsilon^{4}}}\right), \quad \mathbf{X}^{\varepsilon}\left(z_{0}\right)=\mathbf{X}_{\mathrm{ini}},
$$

where $\mathbf{F}^{(q)}: \mathbb{R}^{N^{2}} \rightarrow \mathbb{R}^{N^{2}}$ and $\mathbf{G}^{(q)}: \mathbb{R}^{N^{2}} \rightarrow \mathbb{R}^{N^{2}}$ are smooth functions and $\alpha^{(q)} \in \mathbb{R} \backslash\{0\}$ are distinct along with their sums and differences. Then $\mathbf{X}^{\varepsilon}$ converges to $\mathbf{X}$ the solution of the effective equation

$$
\frac{d \mathbf{X}}{d z}=\mathbf{F}^{(0)}(\mathbf{X})+\sum_{q=1}^{Q} \frac{i}{\alpha^{(q)}}\left[\mathbf{F}^{(q)}(\mathbf{X}), \mathbf{G}^{(q)}(\mathbf{X})\right], \quad \mathbf{X}\left(z_{0}\right)=\mathbf{X}_{\mathrm{ini}}
$$

where $[\cdot, \cdot]$ stands for the Lie brackets defined by

$$
[\mathbf{F}(\mathbf{X}), \mathbf{G}(\mathbf{X})]=\sum_{j, k=1}^{N}\left(\frac{\partial \mathbf{F}(\mathbf{X})}{\partial X_{j k}} G_{j k}(\mathbf{X})-\frac{\partial \mathbf{G}(\mathbf{X})}{\partial X_{j k}} F_{j k}(\mathbf{X})\right) .
$$

Note that, when $\mathbf{F}(\mathbf{X})=\mathbf{A X}$ and $\mathbf{G}(\mathbf{X})=\mathbf{B X}$ where $\mathbf{A}$ and $\mathbf{B}$ are two $N \times N$ matrices, then

$$
[\mathbf{F}(\mathbf{X}), \mathbf{G}(\mathbf{X})]=(\mathbf{A B}-\mathbf{B A}) \mathbf{X} .
$$

Proof. The result can be obtained by a multi-scale expansion ansatz in powers of harmonic phases: 


$$
\begin{aligned}
\mathbf{X}^{\varepsilon}(z)= & \mathbf{X}^{(0)}(z)+\varepsilon^{2} \sum_{q=1}^{Q}\left(\mathbf{X}^{(q, 1)}(z) e^{i \frac{\alpha^{(q) z}}{\varepsilon^{4}}}+\mathbf{X}^{(q,-1)}(z) e^{-i \frac{\alpha^{(q) z}}{\varepsilon^{4}}}\right) \\
& +\varepsilon^{4} \sum_{q=1}^{Q}\left(\mathbf{X}^{(q, 2)}(z) e^{2 i \frac{\alpha^{(q)} z}{\varepsilon^{4}}}+\mathbf{X}^{(q, 0)}(z)+\mathbf{X}^{(q,-2)}(z) e^{-2 i \frac{\alpha^{(q) z} z}{\varepsilon^{4}}}\right) \\
& +\varepsilon^{4} \sum_{1 \leq q<q^{\prime} \leq Q}\left(\mathbf{X}^{\left(q, q^{\prime}, 1,1\right)}(z) e^{i \frac{\left(\alpha(q)+\alpha\left(q^{\prime}\right) z z\right.}{\varepsilon^{4}}}+\mathbf{X}^{\left(q, q^{\prime}, 1,-1\right)}(z) e^{i \frac{\left(\alpha^{(q)}-\alpha^{\left(q^{\prime}\right)}\right) z}{\varepsilon^{4}}}\right) \\
& +\varepsilon^{4} \sum_{1 \leq q<q^{\prime} \leq Q}\left(\mathbf{X}^{\left(q, q^{\prime},-1,1\right)}(z) e^{i \frac{\left(-\alpha(q)+\alpha\left(q^{\prime}\right)\right) z}{\varepsilon^{4}}}+\mathbf{X}^{\left(q, q^{\prime},-1,-1\right)}(z) e^{i \frac{\left(-\alpha(q)-\alpha\left(q^{\prime}\right) z z\right.}{\varepsilon^{4}}}\right)+\cdots
\end{aligned}
$$

Recall that $\alpha^{(q)}$ along with their sums and differences are distinct so that these constitute different harmonics. We now substitute the ansatz (C.2) in (C.1). By subsequently collecting the terms of order $0\left(\varepsilon^{-2}\right)$ and $0\left(\varepsilon^{0}\right)$ and identifying the terms with the same harmonic (i.e. the same rapid phase), we obtain:

$$
\begin{aligned}
& -i \alpha^{(q)} \mathbf{X}^{(q,-1)}=\mathbf{G}^{(q)}\left(\mathbf{X}_{0}\right), \quad i \alpha^{(q)} \mathbf{X}^{(q, 1)}=\mathbf{F}^{(q)}\left(\mathbf{X}_{0}\right), \\
& \frac{d \mathbf{X}^{(0)}}{d z}=\mathbf{F}^{(0)}\left(\mathbf{X}^{(0)}\right)+\sum_{q=1}^{Q} \sum_{j, k} \frac{\partial \mathbf{F}^{(q)}\left(\mathbf{X}^{(0)}\right)}{\partial X_{j k}} X_{j k}^{(q,-1)}+\sum_{j, k} \frac{\partial \mathbf{G}^{(q)}\left(\mathbf{X}^{(0)}\right)}{\partial X_{j k}} X_{j k}^{(q, 1)}, \\
& -2 i \alpha^{(q)} \mathbf{X}^{(q,-2)}=\sum_{j, k} \frac{\partial \mathbf{G}^{(q)}\left(\mathbf{X}^{(0)}\right)}{\partial X_{j k}} X_{j k}^{(q,-1)}, \quad 2 i \alpha^{(q)} \mathbf{X}^{(q, 2)}=\sum_{j, k} \frac{\partial \mathbf{F}^{(q)}\left(\mathbf{X}^{(0)}\right)}{\partial X_{j k}} X_{j k}^{(q, 1)}, \\
& i\left(\alpha^{(q)}+\alpha^{\left(q^{\prime}\right)}\right) \mathbf{X}^{\left(q, q^{\prime}, 1,1\right)}=\sum_{j, k} \frac{\partial \mathbf{F}^{(q)}\left(\mathbf{X}^{(0)}\right)}{\partial X_{j k}} X_{j k}^{\left(q^{\prime}, 1\right)}+\sum_{j, k} \frac{\partial \mathbf{F}^{\left(q^{\prime}\right)}\left(\mathbf{X}^{(0)}\right)}{\partial X_{j k}} X_{j k}^{(q, 1)}, \\
& -i\left(\alpha^{(q)}+\alpha^{\left(q^{\prime}\right)}\right) \mathbf{X}^{\left(q, q^{\prime},-1,-1\right)}=\sum_{j, k} \frac{\partial \mathbf{G}^{(q)}\left(\mathbf{X}^{(0)}\right)}{\partial X_{j k}} X_{j k}^{\left(q^{\prime},-1\right)}+\sum_{j, k} \frac{\partial \mathbf{G}^{\left(q^{\prime}\right)}\left(\mathbf{X}^{(0)}\right)}{\partial X_{j k}} X_{j k}^{(q,-1)}, \\
& i\left(\alpha^{(q)}-\alpha^{\left(q^{\prime}\right)}\right) \mathbf{X}^{\left(q, q^{\prime}, 1,-1\right)}=\sum_{j, k} \frac{\partial \mathbf{F}^{(q)}\left(\mathbf{X}^{(0)}\right)}{\partial X_{j k}} X_{j k}^{\left(q^{\prime},-1\right)}+\sum_{j, k} \frac{\partial \mathbf{G}^{\left(q^{\prime}\right)}\left(\mathbf{X}^{(0)}\right)}{\partial X_{j k}} X_{j k}^{(q, 1)}, \\
& i\left(-\alpha^{(q)}+\alpha^{\left(q^{\prime}\right)}\right) \mathbf{X}^{\left(q, q^{\prime},-1,1\right)}=\sum_{j, k} \frac{\partial \mathbf{G}^{(q)}\left(\mathbf{X}^{(0)}\right)}{\partial X_{j k}} X_{j k}^{\left(q^{\prime}, 1\right)}+\sum_{j, k} \frac{\partial \mathbf{F}^{\left(q^{\prime}\right)}\left(\mathbf{X}^{(0)}\right)}{\partial X_{j k}} X_{j k}^{(q,-1)}
\end{aligned}
$$

Substituting the expressions of $\mathbf{X}^{(q, 1)}$ and $\mathbf{X}^{(q,-1)}$ given by the first equation into the second one gives the desired result. The other equations give the expressions of the corrective terms.

\section{References}

[1] Achenbach, J.D.: Wave propagation in elastic solids. North-Holland, Amsterdam (1973)

[2] Bailly, F., Clouet, J.-F., Fouque, J.-P.: Parabolic and white noise approximation for waves in random media. SIAM J. Appl. Math. 56, 1445-1470 (1996)

[3] Bellman, R., Wing, G.M.: Introduction to invariant imbedding. Wiley, New York (1975)

[4] Blomgren, P., Papanicolaou, G., Zhao, H.: Super-resolution in time-reversal acoustics. J. Acoust. Soc. Am. 111, 230-248 (2002)

[5] Claerbout, J.F.: Imaging the Earths interior. Blackwell Scientific Publications, Palo Alto (1985)

[6] Corones, J., DeFacio, B., Krueger, R.J.: Parabolic approximations to the time-independent elastic wave equations. J. Math. Phys. 23, 577-586 (1982)

[7] Dawson, D., Papanicolaou, G.: A random wave process. Appl. Math. Optim. 12, 97-114 (1984) 
[8] de Hoop, M.V., de Hoop, A.T.: Elastic wave up/down decomposition in inhomogeneous and anisotropic media: an operator approach and its approximations. Wave Motion 20, 57-82 (1994)

[9] Fannjiang, A.: Self-averaging scaling limits for random parabolic waves. Arch. Rational Mech. Anal. 175, 343-387 (2005)

[10] Fannjiang, A., Sølna, K.: Propagation and time-reversal of wave beams in atmospheric turbulence. SIAM Multiscale Model. Simul. 3, 522-558 (2005)

[11] Fouque, J.-P., Garnier, J., Papanicolaou, G., Sølna, K.: Wave propagation and time reversal in randomly layered media. Springer, New York (2007)

[12] Fouque, J.-P, Papanicolaou, G., Samuelides, Y.: Forward and Markov approximation: the strong-intensity-fluctuations regime revisited. Waves in Random Media 8, 303-314 (1998)

[13] Garnier, J., Sølna, K.: Effective transport equations and enhanced backscattering in random waveguides. SIAM J. Appl. Math. 68, 1574-1599 (2008)

[14] Garnier, J., Sølna, K.: Coupled paraxial wave equations in random media in the white-noise regime. To appear in Ann. Appl. Probab. (2008). Available via http://www.proba.jussieu.fr/ garnier/

[15] Haines, A.J.: A phase-front method - I. Narrow-frequency SH waves. Geophys. J. Roy. Astron. Soc. 72, 783-808 (1983)

[16] Huang, K., Papanicolaou, G., Sølna, K., Tsogka, C., Zhao, H.: Efficient numerical simulation for long range wave propagation. J. Comput. Phys. 215, 448-464 (2005)

[17] Huang, K., Sølna, K., Zhao, H.: Coupled parabolic equations for wave propagation. Methods and Applications of Analysis $11,399-412(2004)$

[18] Hudson, J.A.: A parabolic approximation for elastic waves. Wave Motion 2, 207-214 (1980)

[19] Kohler, W., Papanicolaou, G., White, B.: Localization and mode conversion for elastic waves in randomly layered media I. Wave Motion 23, 1-22 (1996)

[20] Kohler, W., Papanicolaou, G., White, B.: Localization and mode conversion for elastic waves in randomly layered media II. Wave Motion 23, 181-201 (1996)

[21] Kurzweil, J., Jarník, J.: Limit processes in ordinary differential equations. Journal of Applied Mathematics and Physics $38,241-256(1987)$

[22] Landers, T., Claerbout, J.F.: Numerical calculations of elastic waves in laterally inhomogeneous media. J. Geophys. Res. $77,1476-1482(1972)$

[23] Liu, W.: Averaging theorems for highly oscillatory differential equations and iterated Lie brackets. SIAM J. Control Optim. 35, 1989-2020 (1997)

[24] Lu, Y.Y., McLaughlin, J.R.: The Riccati method for the Helmholtz equation. J. Acoust. Soc. Am. 100, 1432-1446 (1996)

[25] McCoy, J.J.: A parabolic theory of stress wave propagation through inhomogeneous linearly elastic solids. J. Appl. Mech. 44, 462-468 (1977)

[26] Papanicolaou G., Ryzhik L., and Sølna K., Statistical stability in time reversal, SIAM J. Appl. Math. 64, 1133-1155 (2004)

[27] Papanicolaou, G., Ryzhik, L., Sølna, K.: Self-averaging from lateral diversity in the Itô-Schrödinger equation. SIAM Multiscale Model. Simul. 6, 468-492 (2007)

[28] Ryzhik, L., Keller, J., Papanicolaou, G.: Transport equations for elastic and other waves in random media. Wave Motion 24, 327-370 (1996)

[29] Sølna, K., Papanicolaou, G.: Ray theory for a locally layered medium. Waves in Random Media 10, 151-198 (2000)

[30] Strohbehn, J.W. (ed.): Laser beam propagation in the atmosphere. Springer, Berlin (1978)

[31] Tappert, F.: The parabolic approximation method. In: Wave propagation and underwater acoustics, Keller, J.B., Papadakis, J.S. (eds.), pp. 224-287, Springer, Berlin (1977)

[32] Ursin, B.: Review of elastic and electromagnetic wave propagation in horizontally layered media. Geophysics 48, 1063-1081 (1983)

[33] Wales, S.C., McCoy, J.J.: A comparison of parabolic wave theories for linearly elastic solids. Wave Motion 5, 99-113 (1983) 